\title{
Cadmium stress related to root-to-shoot communication depends on ethylene and auxin in tomato plants
}

\author{
Letícia Rodrigues Alves ${ }^{a}$, Carolina Cristina Monteiro ${ }^{a}$, Rogério Falleiros Carvalho ${ }^{a}$, \\ Patricia Cury Ribeiro ${ }^{\mathrm{a}}$, Tiago Tezotto ${ }^{\mathrm{b}}$, Ricardo Antunes Azevedo ${ }^{\mathrm{c}}$, \\ Priscila Lupino Gratão ${ }^{\mathrm{a}, *}$ \\ ${ }^{a}$ UNESP - Univ. Estadual Paulista, Faculdade de Ciências Agrárias e Veterinárias-FCAV, Depto. de Biologia Aplicada à Agropecuária (DBAA), 14884-900 \\ Jaboticabal, SP, Brazil \\ ${ }^{\mathrm{b}}$ Unifeob - Centro Universitário da Fundação de Ensino Octávio Bastos, 13874-149, São João da Boa Vista, SP, Brazil \\ ${ }^{\mathrm{c}}$ USP - Universidade de São Paulo, Escola Superior de Agricultura Luiz de Queiroz-ESALQ Depto. de Genética, 13418-900 Piracicaba, SP, Brazil
}

\section{A R T I C L E I N F O}

\section{Article history:}

Received 17 August 2016

Received in revised form 14 November 2016

Accepted 19 November 2016

Available online 23 November 2016

\section{Keywords:}

Antioxidant defence

Diageotropica

Never ripe

Micro-Tom

Oxidative stress

Reactive oxygen species

\begin{abstract}
A B S T R A C T
Stress perception and signalling pathways between plant organs involve complex mechanisms and remain a major focus of interest. To further address the role of phytohormones in the modulation of stress perception from root-to-shoot signalling, we used ethylene-insensitive Never ripe ( $\mathrm{Nr}$ ) and auxininsensitive diageotropica (dgt) tomato mutants combined with the grafting technique. Lipid peroxidation, $\mathrm{H}_{2} \mathrm{O}_{2}$, chlorophyll and proline contents, and activities of superoxide dismutase (SOD), catalase (CAT), ascorbate peroxidase (APX) and glutathione reductase (GR) in non-grafted and grafted tomato mutants subjected to cadmium (Cd) were analysed. The results revealed different responses according to genotype, grafting combination and $\mathrm{Cd}$ application. Non-grafted hormonal mutants exhibited higher $\mathrm{Cd}$ content in roots than MT plants, being 39.9\% in $\mathrm{Nr}$ and $17.7 \%$ in $d g t$ plants, whereas in leaves, the $\mathrm{Cd}$ content was higher in $\mathrm{Nr}$ plants. In grafted plants, where the rootstocks were exposed to $\mathrm{Cd}$ before grafting, the MT rootstock exhibited the highest $\mathrm{Cd}$ content. In non-grafted plants following $\mathrm{Cd}$ application, roots of $\mathrm{Nr}$ also exhibited a decrease in Ca concentration, whilst $\mathrm{Mg}, \mathrm{S}$, Cu and $\mathrm{Zn}$ decreased in $\mathrm{Nr}$ leaves. In grafted plants, it was possible to notice peculiar differences in nutrient concentration patterns according to grafting combination and $\mathrm{Cd}$ application. The proline and chlorophyll contents were less affected in the hormonal mutants. In the presence of $\mathrm{Cd}$, the scions of grafted plants exhibited increased antioxidant enzymes activities in response to a signal from the rootstocks. However, it was possible to associate the involvement of ethylene and auxin with the antioxidant responses because the $\mathrm{Nr}$ and $d g t$ genotypes were less affected by Cd stress than their wild-type counterpart, MT.
\end{abstract}

(c) 2016 Elsevier B.V. All rights reserved.

\section{Introduction}

Cadmium (Cd) is a dangerous heavy metal that is toxic to many organisms. This metal can accumulate in the environment as a result of anthropogenic activities and its main sources are herbicides, pesticides, chemical fertilizers, irrigation with contaminated water and pollutants from industrial processes (Hédiji et al., 2015; Zouari et al., 2016a). Although Cd is a nonessential element, it can be easily taken up by plants, causing morphological, structural, biochemical, physiological dysfunctions and alteration of transcript profile (Gratão et al., 2009; Polle et al., 2013; Ahmad

\footnotetext{
* Corresponding author.

E-mail address: plgratao@fcav.unesp.br (P.L. Gratão).
}

et al., 2016; Luo et al., 2016). Even at low concentrations, Cd can induce or stimulate uncontrolled oxidation and cause alterations in cell homeostasis and electrolyte leakage, which trigger biochemical responses against oxidative stress, inducing a wide range of antioxidant defence systems (Iannone et al., 2010; He et al., 2013a,b; Anjum et al., 2015; Zouari et al., 2016b).

Uncontrolled oxidation can be detoxified by complex enzymatic and non-enzymatic mechanisms that interact in an attempt to minimize oxidative stress damage and maintain the cell redox state (Zhang et al., 2014; Gratão et al., 2015). The primary defence step at the cellular level comprises antioxidant enzymes such as superoxide dismutase (SOD, EC 1.15.1.1), which converts $\mathrm{O}_{2}{ }^{-}-$to $\mathrm{H}_{2} \mathrm{O}_{2}$ (Noctor and Foyer, 2016). Subsequently, $\mathrm{H}_{2} \mathrm{O}_{2}$ may be detoxified to $\mathrm{H}_{2} \mathrm{O}$ by ascorbate peroxidase (APX, EC 1.11.1.11), catalase (CAT, EC 1.11.1.6) and glutathione peroxidase (GPX, EC 
1.11.1.9) (Roychoudhury et al., 2012; Hippler et al., 2015; Liu et al., 2015; Khaliq et al., 2015), among other peroxidases.

In addition, non-enzymatic mechanisms including compounds such as proline, flavonoids, carotenoids, ascorbate and glutathione (GSH) may also be responsible for quenching excessive reactive oxygen species (ROS) (Wu et al., 2007; Ferraz et al., 2012; He et al., 2015; Zouari et al., 2016a,b). The regeneration of GSH formed from oxidized glutathione (GSSG) is catalysed by glutathione reductase (GR, EC 1.6.4.2) using NADPH as a reducing agent.

Plant-stress mechanisms may require the interplay between interconnected networks of cellular responses and signalling molecules under Cd stress conditions (Chmielowska-Bak et al., 2014; Islam et al., 2015; Shi et al., 2015). For instance, phytohormones can also participate and interact with redox signalling to control responses to abiotic stresses (Gratão et al., 2012; Carvalho et al., 2013; Bankaji et al., 2014). Nonetheless, the mechanisms involving the interaction between phytohormones and stress responses are still poorly understood, especially the cross talk involved in root-to-shoot communication.

Special attention should be given to ethylene, which can be considered a 'stress hormone' involved in multiple molecular and physiological plant responses and which regulates various growth and cellular defence mechanisms in response to toxic metals (Schellingen et al., 2014; Van de Poel et al., 2015). This implies a synergistic effect between the biosynthesis of ROS and ethylene (Djanaguiraman et al., 2009). Although the molecular relationship between ethylene biosynthesis and $\mathrm{Cd}$ stress has not been well established, ethylene may contribute to the regulation of the early Cd-induced oxidative challenge via the control of the GSH content and the expression of signalling genes (Schellingen et al., 2015). Therefore, answers are still needed with respect to the mechanisms underlying ethylene regulation of plant response to metal stress and subsequent effects on plant sensitivity or tolerance (Asgher et al., 2015). Studies have shown that ethylene-insensitive mutants are less sensitive to heavy metal stress conditions (Bueso et al., 2007; Gratão et al., 2012).

In addition to ethylene, the phytohormone auxin may also be involved in stress signalling and defence responses (George et al., 2010), e.g., heavy metal tolerance and accumulation (Fassler et al., 2010; Keunen et al., 2016). Current research has shown that auxin may induce morphogenic responses (Potters et al., 2009; Monteiro et al., 2012), which may prevent adverse effects of environmental stresses (Tognetti et al., 2011). In contrast, the suppression of auxin may improve stress tolerance by mediated growth suppression and reallocation of metabolic resources to resistance establishment (Park et al., 2007).

Although multiple stress responses are essential for plant survival under heavy metal stress conditions, the exact roles of ethylene and auxin phytohormones in these responses are not understood. Therefore, the use of hormonal mutants is a powerful tool to study hormonal modulation, providing insights into the cross-talk between ROS signalling and phytohormones under stress conditions. In tomato (Solanum lycopersicum), the ethylene LeETR3 receptor corresponds to the mutation Never ripe $(\mathrm{Nr})$ (Wilkinson et al., 1995), which does not respond to either endogenously generated or exogenous ethylene (Lanahan et al., 1994). The diageotropica (dgt) tomato mutant exhibits a considerably reduced sensitivity to the hormone auxin (Kelly and Bradford, 1986), related to the DIAGEOTROPICA (DGT) gene, which encodes a component of the specific auxin-signalling pathway (Retzer and Luschnig, 2015) and regulates auxin transport in lateral root formation (Ivanchenko et al., 2015).

Our prior studies with $\mathrm{Nr}$ and $d g t$ tomato mutants (Monteiro et al., 2011; Gratão et al., 2012) revealed the involvement of ethylene and auxin in antioxidant mechanisms. For example, $\mathrm{Nr}$ genotype was shown to be more affected than $d g t$ plants by the $\mathrm{Cd}-$ imposed stress because $\mathrm{Nr}$ retains a partial sensitivity to ethylene (Castagna et al., 2007), whilst dgt may withstand or avoid stress imposed by $\mathrm{Cd}$, due to the fact that the known auxin-stimulated ethylene production is compromised in $d g t$ plants (Gratão et al., 2012). In another recent study, the tomato sitiens ABA-deficient mutant (sit) exposed to $\mathrm{Cd}$ has also shown a number of antioxidant responses, which helped to unravel the relative importance of $A B A$ in regulating cell responses to stressful conditions induced by $\mathrm{Cd}$ (Pompeu et al., 2017). With these results, new questions arise regarding the mechanisms connecting antioxidant responses, ROS and signalling triggered by plant hormones in communication with plant organs.

The grafting technique can be an elegant mechanism to demonstrate root-to-shoot and shoot-to-root communication (Molnar et al., 2010). This is because the plant vascular system can serve as a conduit for the transport of long-distance nutrients and signalling molecule inter-organ communication (Spiegelman et al., 2013). Therefore, the use of grafting can be an interesting tool to study the effect of metals on plant development, in particular the antioxidant stress response. In another recent study in our group, the use of grafted tomato plants revealed distinct trends in crosstalk between antioxidant responses and signalling of plant organs during stress (root-to-shoot) that clearly indicated signalling responses from the rootstocks, allowing sufficient time to activate defence mechanisms in the shoot (Gratão et al., 2015).

In this report, we used the $N r$ and $d g t$ tomato mutants and the grafting technique with the objective to gain a better understanding or to provide more information on the modulation of interorgan oxidative stress with a focus on the antioxidant systems and the roles of auxin and ethylene under Cd stress conditions.

\section{Materials and methods}

\subsection{Plant material and growth conditions}

Seeds of the tomato (S. lycopersicum L.) cultivar Micro-Tom (MT) and the mutants diageotropica (dgt) and Never ripe $(\mathrm{Nr})$ were sterilized using a sodium hypochlorite solution (5\%), rinsed three times and sown in boxes filled with a mixture of 1:1 (by volume) commercial pot mix (Plantmax HT Eucatex, Brazil) and vermiculite supplemented with $1 \mathrm{~g} \mathrm{~L}^{-1}$ 10:10:10 NPK (nitrogen-phosphoruspotassium) and $4 \mathrm{gL}^{-1}$ lime $\left(\mathrm{MgCO}_{3}+\mathrm{CaCO}_{3}\right)$. The boxes were maintained in a greenhouse with an average mean temperature of $23.2^{\circ} \mathrm{C}$, relative humidity $77.6 \%$, and an $11.8 \mathrm{~h}$ photoperiod (autumn). When two true leaves were completely formed, two seedlings were transplanted to $0.350 \mathrm{~L}$ Leonard pots (Vincent, 1975 ) containing sterilized sand, polystyrene (4:3) and modified Hoagland's nutrient solution $(250 \mathrm{~mL})$. After seedling establishment, only one plant was maintained per pot. Thirty five-day-old plants were further grown in the same solution with $0 \mathrm{mM}$ or $1 \mathrm{mM}$ $\mathrm{CdCl}_{2}$ (Gratão et al., 2015), which was changed weekly.

Forty five-day-old plants were grafted using the cleft method, and the plant was cut off approximately $3 \mathrm{~cm}$ above soil level; this part was used as the rootstock, and a portion of the shoot was used as scion. The scion was inserted into the slit in the centre of the rootstock stem, and the joined parts were tied firmly using $5-\mathrm{cm}-$ wide plastic film and a spring clip. When the grafting was completed, all seedlings were covered with transparent plastic bags and a shade net. The transparent plastic films were maintained for four days to ensure high relative humidity. The plastic binding films were moistened daily and removed when the graft union was formed. Germinating buds of rootstocks were also removed daily. The rootstocks had Cd exposure blocked at the time of grafting. The establishment of the scion-rootstock and the nongrafted plants are presented in Fig. 1. 


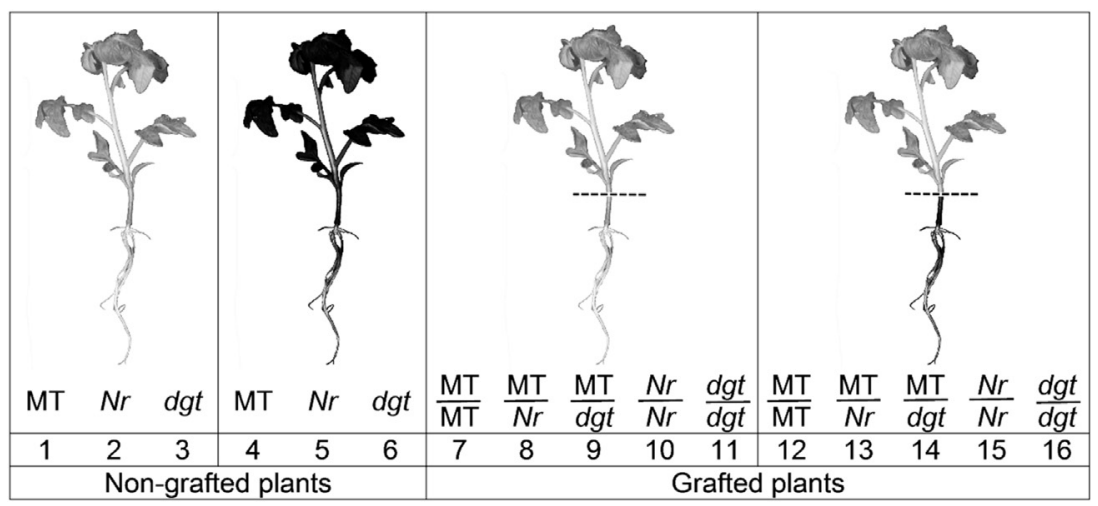

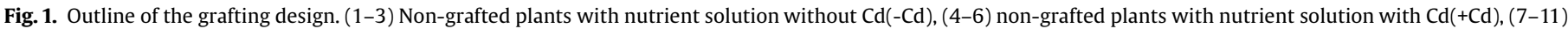
grafted plants: $-\mathrm{Cd} /-\mathrm{Cd}=$ scion/rootstock, nutrient solution without $\mathrm{Cd},(12-16)$ grafted plants: $-\mathrm{Cd} /+\mathrm{Cd}=\mathrm{scion} / \mathrm{rootstock}$, nutrient solution with $\mathrm{Cd}$.

After a period of 75 days post germination, corresponding to 45 days of exposure to $\mathrm{CdCl}_{2}$ and 30 days after grafting, samples of roots and leaves (excluding petioles) were harvested, rinsed and immediately immersed in liquid $\mathrm{N}_{2}$ and stored at $-80^{\circ} \mathrm{C}$ for further analysis of lipid peroxidation, $\mathrm{H}_{2} \mathrm{O}_{2}$ content, chlorophyll determination, proline content, enzyme extraction and protein determination; samples of roots and leaves were kept in paper bags and dried in a drying oven $\left(60^{\circ} \mathrm{C}\right)$ until constant weight for dry mass determination, $\mathrm{Cd}$ content and nutritional analysis.

\subsection{Cd content and nutritional analysis}

Dry root and leaves samples (200.0 $\mathrm{mg} \mathrm{DW}$ ) were milled and microwave digested with $2 \mathrm{~mL} 70 \% \mathrm{HNO}_{3}, 2 \mathrm{~mL} \mathrm{H}_{2} \mathrm{O}_{2}$ and $2 \mathrm{~mL}$ Milli-Q water $\left(18.2 \mathrm{MX} \mathrm{cm} \mathrm{a} 25^{\circ} \mathrm{C}\right)$ at a controlled pressure of $2 \mathrm{MPa}$ (Chilimba et al., 2011). The digested samples were diluted to $10 \mathrm{~mL}$ with Milli-Q water and stored. The $\mathrm{Cd}$ content was determined using ICP-OES (Jobin Yvon, JY50P Longjumeau, France). The $\mathrm{P}, \mathrm{K}, \mathrm{Ca}, \mathrm{Mg}, \mathrm{Cu}, \mathrm{Fe}, \mathrm{Mn}$ and $\mathrm{Zn}$ contents were determined by X-ray fluorescence (EDXRF) analysis, using samples prepared as loose powder, as described by Tezotto et al. (2013).

\subsection{Lipid peroxidation}

Lipid peroxidation was measured by estimating the content of thiobarbituric acid reactive substances (TBARS) as described by Heath and Packer (1968). Plant tissue was ground with 20\% (w/v) polyvynilpyrrolidone (PVPP) and $0.1 \%$ trichloroacetic acid (TCA). After centrifugation at $11,000 \times g$ for $10 \mathrm{~min}$, the supernatant was added to a solution of $20 \%$ TCA and $5 \%$ thiobarbituric acid (TBA) and incubated in a water bath at $95^{\circ} \mathrm{C}$ for $30 \mathrm{~min}$. The reaction was stopped by cooling in an ice bath for $10 \mathrm{~min}$ and centrifuged at $11,000 \times \mathrm{g}$ for $10 \mathrm{~min}$. The concentration of malondialdehyde (MDA) equivalents was determined spectrophotometrically between 535 and $600 \mathrm{~nm}$; data were calculated using an extinction coefficient of $1.55 \times 10^{-5} \mathrm{~mol}^{-1} \mathrm{~cm}^{-1}$ (Gratão et al., 2012).

\section{4. $\mathrm{H}_{2} \mathrm{O}_{2}$ content}

The $\mathrm{H}_{2} \mathrm{O}_{2}$ content was estimated following the method of Alexieva et al. (2001). Plant tissues were homogenized in thiobarbituric acid (0.1\%) and centrifuged at $10,000 \times g$ for $10 \mathrm{~min}$. The supernatant was added to $100 \mathrm{mM}$ potassium phosphate buffer $(\mathrm{pH} 7.50)$ and $1 \mathrm{M}$ potassium iodide solution. This solution was incubated on ice for one hour, the absorbance was read at $390 \mathrm{~nm}$, and the $\mathrm{H}_{2} \mathrm{O}_{2}$ content was determined using a known $\mathrm{H}_{2} \mathrm{O}_{2}$ concentration curve as a standard.

\subsection{Chlorophyll determination}

Fresh leaves $(0.50 \mathrm{mg})$ were added in tube with $2 \mathrm{~mL}$ acetone (100\%). After shaking for $72 \mathrm{~h}$ at $60 \times \mathrm{g}$ at $4{ }^{\circ} \mathrm{C}$ the sample was read spectrophotometrically in $470 \mathrm{~nm}$ for carotenoids and $645 \mathrm{~nm}$ and $662 \mathrm{~nm}$ for chlorophyll a and b, respectively. The chlorophyll and content was calculated as described by Lichtentlaler (1987).

\subsection{Proline content}

The proline content was determined spectrophotometrically as described by Bates et al. (1973). Leaves and root samples were homogenized in $3 \%$ sulphosalicylic acid. The mixture filtrate was reacted with $1 \mathrm{~mL}$ each of acid ninhydrin and glacial acetic acid and was placed in boiling waterfor $1 \mathrm{~h}$. Toluene $(4 \mathrm{~mL})$ was added to the mixture, and the absorbance was measured at $520 \mathrm{~nm}$ and calculated as $\mathrm{mmol} \mathrm{g}^{-1}$ fresh weight against standard proline.

\subsection{Enzyme extraction and protein determination}

Fresh leaf tissue was homogenized in a chilled mortar with a pestle using an extraction buffer containing $100 \mathrm{mM}$ potassium phosphate buffer ( $\mathrm{pH} 7.5$ ), $1 \mathrm{mM}$ ethylenediamine tetraacetic acid (EDTA), $3 \mathrm{mM}$ DL-dithiothreitol and 5\% (w/v) insoluble polyvinylpolypyrrolidone (Boaretto et al., 2014) in 3:1 vol/fresh weight ratio. The homogenate was centrifuged at $10,000 \times \mathrm{g}$ for $30 \mathrm{~min}$, and the supernatant was stored at $-80^{\circ} \mathrm{C}$ for further determination of SOD, CAT, GR and APX activities. The protein concentration was determined following the method of Bradford (1976) using bovine serum albumin as a standard.

\subsection{Superoxide dismutase assay}

SOD activity was determined by activity staining using nondenaturing PAGE as described by Azevedo et al. (1998). Plant extracts were submitted to non-denaturing-PAGE separation: the gels were rinsed in distilled-deionized water and maintained in the dark for $30 \mathrm{~min}$ in $50 \mathrm{mM}$ potassium phosphate buffer $(\mathrm{pH} 7.8$ ) containing $0.05 \mathrm{mM}$ riboflavin, $0.1 \mathrm{mM}$ nitro blue tetrazolium, 1 mM EDTA, and 0.3\% N,N,N',N'-tetramethylethyllenediamine. The gels were rinsed with distilled-deionized water and then illuminated in water until the achromatic bands of SOD activity were visible on a purple-stained gel. SOD isoenzymes were 
distinguished and classified by their sensitivity to inhibition by $5 \mathrm{mM}$ hydrogen peroxide $\left(\mathrm{H}_{2} \mathrm{O}_{2}\right)$ or $2 \mathrm{mM}$ potassium cyanide (Azevedo et al., 1998).

\subsection{Catalase assay}

Catalase activity (CAT) was assayed spectrophotometrically at $25^{\circ} \mathrm{C}$ in a reaction mixture containing $1 \mathrm{~mL}$ of $100 \mathrm{mM}$ potassium phosphate buffer ( $\mathrm{pH} 7.5$ ) containing $25 \mu \mathrm{L} \mathrm{H}_{2} \mathrm{O}_{2}$ (30\% solution). The activity was determined by monitoring the decomposition of $\mathrm{H}_{2} \mathrm{O}_{2}$ at $240 \mathrm{~nm}$ over $1 \mathrm{~min}$ as described by Nogueirol et al. (2015). CAT activity was expressed as $\mu \mathrm{mol} \mathrm{min}^{-1} \mathrm{mg}^{-1}$ protein.

\subsection{Ascorbate peroxidase assay}

Ascorbate peroxidase (APX) activity was measured spectrophotometrically in a reaction consisting of plant extract, $80 \mathrm{mM}$ potassium phosphate buffer ( $\mathrm{pH} 7.0$ ) containing $5 \mathrm{mM}$ ascorbate, $1 \mathrm{mM}$ EDTA, and $1 \mathrm{mM} \mathrm{H}_{2} \mathrm{O}_{2}$ (Gratão et al., 2012). APX activity was measured by monitoring the rate of ascorbate oxidation at $290 \mathrm{~nm}$ at $30^{\circ} \mathrm{C}$. APX activity was expressed as nmol ascorbate $\mathrm{min}^{-1} \mathrm{mg}^{-1}$ protein.

\subsection{Glutathione reductase assay}

Glutathione reductase (GR) was assayed spectrophotometrically at $30^{\circ} \mathrm{C}$ as described in Carvalho et al. (2013). The assay mixture consisted of $100 \mathrm{mM}$ potassium phosphate buffer (pH 7.5) containing $1 \mathrm{mM}$ 5,5 dithiobis (2-nitrobenzoic acid), $1 \mathrm{mM}$ GSSG and $0.1 \mathrm{mM}$ NADPH. The rate of reduction of GSSG was followed by monitoring the increase in absorbance at $412 \mathrm{~nm}$ over $1 \mathrm{~min}$. GR activity was expressed as mmol $\mathrm{min}^{-1} \mathrm{mg}^{-1}$ protein.

\subsection{Statistical analysis}

The experimental design was randomized using six plants per treatment from three replicate pots. The result of each plant was expressed as the mean and standard error of the mean $( \pm$ SEM) of three independent replicates of each extract for plant growth, $\mathrm{Cd}$ content and nutritional analyses, TBARS, chlorophyll and proline contents, CAT, APX and GR activities. The statistical analysis was performed using the Assistat software 7.7 Beta ${ }^{\circledR}$. A multiple comparison between means using Duncan's test was followed by an individual ANOVA for each character at a 0.05 level of significance.

\section{Results}

\subsection{Plant growth and Cd content}

Over a 75 days post germination period, grafted plants exhibited a reduction in dry mass when compared with nongrafted plants. The grafting technique had a negative effect on growth of all genotypes. Rootstock and scion exhibited a drastically reduction in dry mass when compared with roots and leaves of non-grafted plants (Fig. 2). Among non-grafted plants, MT plants cultivated in the presence of $\mathrm{CdCl}_{2}$ exhibited a decrease of $41.5 \%$ in root growth when compared with the MT genotype. The $\mathrm{Nr}$ mutant exhibited a reduction of $58 \%$ in leaf dry mass, whilst for $d g t$ plants there was no alteration when compared with control plants (without $\mathrm{CdCl}_{2}$ ) (Fig. 2). Non-grafted hormonal mutants exhibited higher Cd content in roots than in roots of MT plants, with $39.9 \%$ for

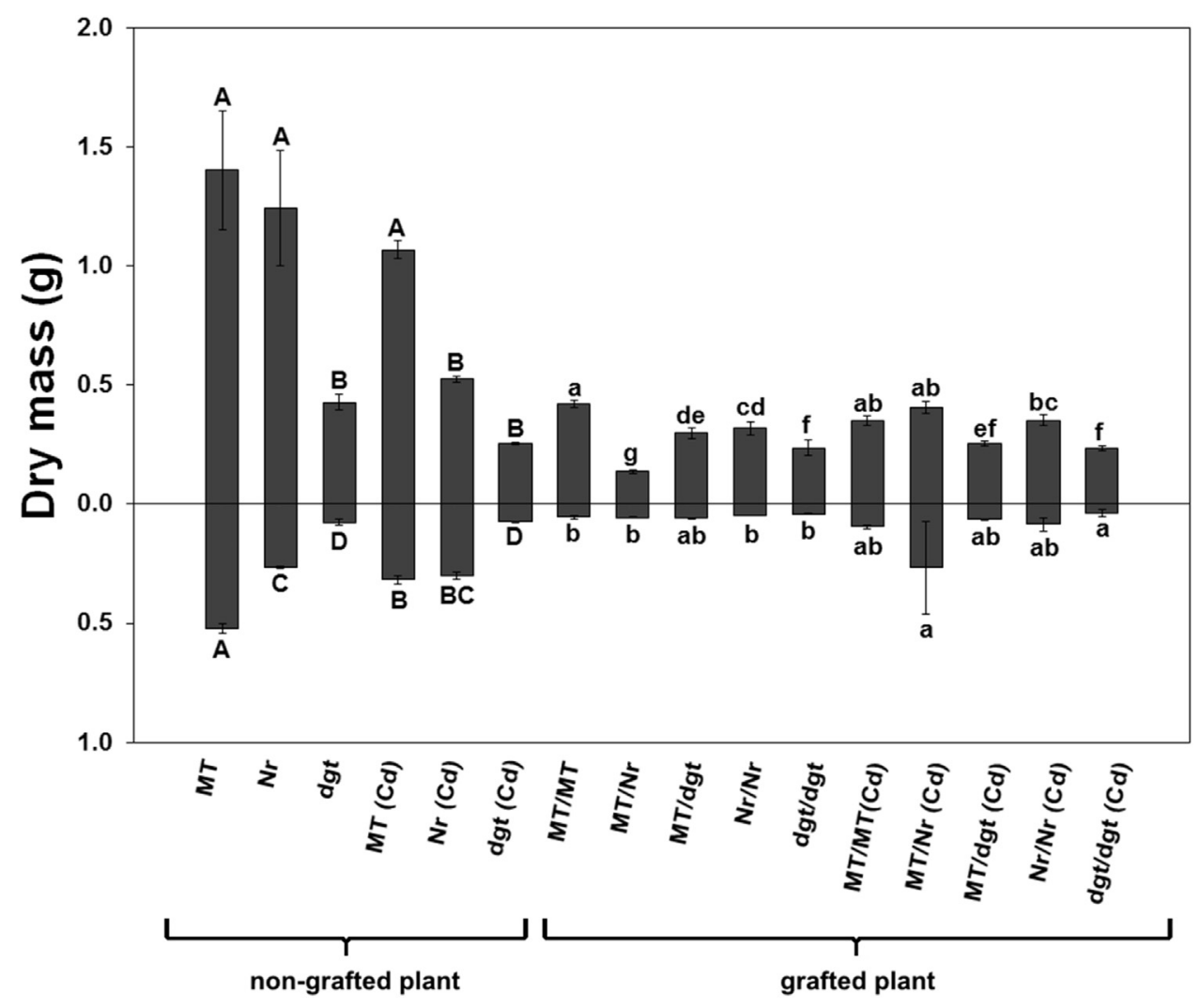

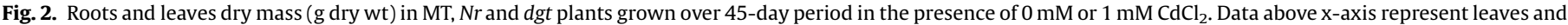

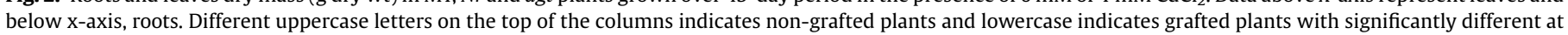
$\mathrm{P}<0.05$ by Duncan test. 
Table 1

$\mathrm{Cd}$ accumulation ( $\mu \mathrm{gg}^{-1}$ dry weight) in MT, $\mathrm{Nr}$ and $d g t$ plants grown over a 45 -day period in the presence of $1 \mathrm{mM} \mathrm{CdCl}$. The $0 \mathrm{mM} \mathrm{CdCl}_{2}$ values were all below $0.6 \mu \mathrm{g} \mathrm{g}^{-1}$ dry weight. Different uppercase letters indicates non-grafted plants and lowercase indicates grafted plants with significantly different at $\mathrm{P}<0.05$ by Duncan test.

\begin{tabular}{|c|c|c|c|}
\hline & \multicolumn{2}{|c|}{ Treatments } & $\begin{array}{l}\mathrm{Cd} \\
\mu \mathrm{gg}^{-1}\end{array}$ \\
\hline Non-grafted plant & $\begin{array}{l}\text { MT (Cd) } \\
\text { MT (Cd) } \\
\mathrm{Nr}(\mathrm{Cd}) \\
\mathrm{Nr}(\mathrm{Cd}) \\
\operatorname{dgt}(\mathrm{Cd}) \\
\operatorname{dgt}(\mathrm{Cd})\end{array}$ & $\begin{array}{l}\text { leaves } \\
\text { roots } \\
\text { leaves } \\
\text { roots } \\
\text { leaves } \\
\text { roots }\end{array}$ & $\begin{array}{l}492.77 \pm 39.32^{\mathrm{B}} \\
5662.90 \pm 736.52^{\mathrm{C}} \\
931.93 \pm 63.21^{\mathrm{A}} \\
9325.07 \pm 470.69^{\mathrm{A}} \\
407.60 \pm 121.25^{\mathrm{B}} \\
7673.53 \pm 526.29^{\mathrm{B}}\end{array}$ \\
\hline Grafted plant & $\begin{array}{l}\text { MT } \\
\mathrm{MT}(\mathrm{Cd}) \\
\mathrm{MT} \\
\mathrm{Nr}(\mathrm{Cd}) \\
\mathrm{MT} \\
\text { dgt (Cd) } \\
\mathrm{Nr} \\
\mathrm{Nr}(\mathrm{Cd}) \\
\text { dgt } \\
\text { dgt (Cd) }\end{array}$ & $\begin{array}{l}\text { scion } \\
\text { rootstock } \\
\text { scion } \\
\text { rootstock } \\
\text { scion } \\
\text { rootstock } \\
\text { scion } \\
\text { rootstock } \\
\text { scion } \\
\text { rootstock }\end{array}$ & $\begin{array}{l}120.57 \pm 20.73^{\mathrm{a}} \\
1908.43 \pm 121.55^{\mathrm{a}} \\
83.47 \pm 11.58^{\mathrm{a}} \\
879.83 \pm 262.90^{\mathrm{c}} \\
101.30 \pm 12.68^{\mathrm{a}} \\
1468.17 \pm 132.00^{\mathrm{b}} \\
112.33 \pm 10.92^{\mathrm{a}} \\
1029.90 \pm 141.28^{\mathrm{c}} \\
33.07 \pm 14.67^{\mathrm{b}} \\
454.33 \pm 164.58^{\mathrm{d}}\end{array}$ \\
\hline
\end{tabular}

$\mathrm{Nr}$ and $17.7 \%$ for $d g t$ plants, whereas in leaves, the $\mathrm{Cd}$ content was higher in $\mathrm{Nr}$ plants (Table 1). In grafted plants, where the rootstocks were exposed to $\mathrm{Cd}$ before grafting, the MT rootstock exhibited the highest $\mathrm{Cd}$ content, and a small portion of $\mathrm{Cd}$ accumulated in above-ground parts of all combination (Table 1 ).

\subsection{Nutritional analysis}

Macronutrients and micronutrients were taken up and distributed differently among plant genotypes depending on the $\mathrm{Cd}$ exposure treatment and the tissue analysed (Table 2). In nongrafted plants following Cd application, MT roots exhibited a decrease in $\mathrm{Ca}$ concentration, whereas the $\mathrm{P}, \mathrm{Mg}, \mathrm{K}, \mathrm{Cu}$ and $\mathrm{Zn}$ concentrations decreased in MT leaves. Roots of $\mathrm{Nr}$ also exhibited a decrease in Ca concentration, whilst $\mathrm{Mg}, \mathrm{S}, \mathrm{Cu}$ and $\mathrm{Zn}$ decreased in $\mathrm{Nr}$ leaves (Table 2). No changes were observed in roots nutrient concentrations in dgt plants, irrespective of $\mathrm{Cd}$ application. However, leaves of $d g t$ plants exhibited an increased S concentration in the presence of $\mathrm{Cd}$. In grafted plants, it was possible to notice peculiar differences in nutrient concentration patterns according to grafting combination and $\mathrm{Cd}$ application. For instance, MT scions of grafted MT(-Cd)/MT(+Cd) plants did not exhibit differences in nutrient concentration compared with control scions of grafted MT(-Cd)/MT(-Cd) plants. However, scions of grafted MT(-Cd)/MT(+Cd) plants exhibited low $\mathrm{Cu}$ concentrations compared with scions of grafted $\mathrm{MT}(-\mathrm{Cd}) / \operatorname{dgt}(+\mathrm{Cd})$ plants. Moreover, scions of grafted $\mathrm{MT}(-\mathrm{Cd}) / \mathrm{Nr}(+\mathrm{Cd})$ plants exhibited a decreased $\mathrm{S}$ concentration compared with scions of grafted MT $(-\mathrm{Cd}) / \mathrm{MT}(+\mathrm{Cd})$ plants. On the other hand, the $\mathrm{Nr}(-\mathrm{Cd}) / \mathrm{Nr}(+\mathrm{Cd})$ rootstocks exhibited an increase in $\mathrm{Mg}$ concentration compared with MT(-Cd)/Nr(+Cd) rootstocks, whilst grafted MT(-Cd) $/ N r(+\mathrm{Cd})$ and $N r(-\mathrm{Cd}) / N r(+\mathrm{Cd})$ rootstocks exhibited an increase in $\mathrm{Mn}$. Moreover, rootstocks of grafted $d g t(-\mathrm{Cd}) / \operatorname{dg} t(+\mathrm{Cd})$ plants exhibited an increased $P$ concentration compared with rootstocks of grafted MT(-Cd)/dgt(+Cd) plants (Table 2$)$.

Table 2

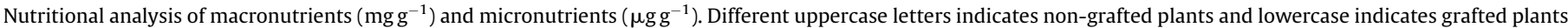
with significantly different at $\mathrm{P}<0.05$ by Duncan test.

\begin{tabular}{|c|c|c|c|c|c|c|c|c|c|c|c|}
\hline & Treatments & & $\begin{array}{l}\mathrm{P} \\
\mathrm{mg} \mathrm{g}^{-1}\end{array}$ & $\mathrm{Ca}$ & $\mathrm{Mg}$ & $\mathrm{S}$ & K & $\begin{array}{l}\mathrm{Cu} \\
\mu \mathrm{gg}^{-1}\end{array}$ & $\mathrm{Fe}$ & $\mathrm{Mn}$ & $\mathrm{Zn}$ \\
\hline \multirow{12}{*}{$\begin{array}{l}\text { Non-grafted } \\
\text { plant }\end{array}$} & MT & leaves & $4.4 \pm 0.5^{\mathrm{AB}}$ & $11.6 \pm 0.3^{A}$ & $3.4 \pm 0.1^{\mathrm{A}}$ & $3.9 \pm 0.2^{\mathrm{A}}$ & $18.2 \pm 0.2^{\mathrm{A}}$ & $1.3 \pm 0.1^{\mathrm{A}}$ & $75.7 \pm 3.9^{\mathrm{B}}$ & $43.2 \pm 4.3^{\mathrm{AB}}$ & $2.8 \pm 0.3^{\mathrm{A}}$ \\
\hline & MT & root & $2.0 \pm 0.5^{\mathrm{A}}$ & $6.4 \pm 1.0^{\mathrm{A}}$ & $3.9 \pm 0.9^{\mathrm{AB}}$ & $3.0 \pm 0.4^{\mathrm{A}}$ & $16.1 \pm 1.4^{\mathrm{A}}$ & $3.0 \pm 0.3^{\mathrm{A}}$ & $251.6 \pm 23.5^{\mathrm{A}}$ & $189.0 \pm 55.5^{\mathrm{A}}$ & $22.0 \pm 18.4^{\mathrm{A}}$ \\
\hline & $\mathrm{Nr}$ & leaves & $3.9 \pm 0.4^{\mathrm{AB}}$ & $10.4 \pm 0.4^{\mathrm{AB}}$ & $3.4 \pm 0.1^{\mathrm{A}}$ & $3.4 \pm 0.1^{\mathrm{AB}}$ & $17.8 \pm 0.6^{\mathrm{A}}$ & $1.3 \pm 0.1^{\mathrm{A}}$ & $75.9 \pm 3.1^{\mathrm{B}}$ & $35.7 \pm 4.1^{\mathrm{AB}}$ & $2.6 \pm 0.3^{A}$ \\
\hline & $\mathrm{Nr}$ & root & $1.6 \pm 0.3^{\mathrm{A}}$ & $6.1 \pm 0.2^{\mathrm{A}}$ & $3.6 \pm 0.4^{\mathrm{AB}}$ & $2.9 \pm 0.3^{\mathrm{A}}$ & $11.1 \pm 2.9^{\mathrm{AB}}$ & $3.4 \pm 1.2^{\mathrm{A}}$ & $330.1 \pm 23.5^{\mathrm{A}}$ & $257.6 \pm 74.4^{\mathrm{A}}$ & $19.0 \pm 10.8^{\mathrm{A}}$ \\
\hline & dgt & leaves & $5.3 \pm 0.2^{\mathrm{A}}$ & $11.1 \pm 0.4^{\mathrm{A}}$ & $3.6 \pm 0.1^{\mathrm{A}}$ & $3.3 \pm 0.1^{\mathrm{B}}$ & $16.2 \pm 0.4^{\mathrm{AB}}$ & $1.3 \pm 0.0^{\mathrm{A}}$ & $106.1 \pm 14.3^{\mathrm{A}}$ & $24.4 \pm 1.1^{\mathrm{B}}$ & $1.4 \pm 0.1^{\mathrm{B}}$ \\
\hline & dgt & root & $1.5 \pm 0.5^{\mathrm{A}}$ & $6.0 \pm 0.6^{\mathrm{A}}$ & $3.6 \pm 0.2^{\mathrm{AB}}$ & $2.5 \pm 0.7^{\mathrm{A}}$ & $12.1 \pm 1.6^{\mathrm{AB}}$ & $2.4 \pm 1.1^{\mathrm{A}}$ & $394.8 \pm 43.8^{A}$ & $178.0 \pm 47.0^{\mathrm{A}}$ & $32.2 \pm 30.2^{\mathrm{A}}$ \\
\hline & $\mathrm{MT}(\mathrm{Cd})$ & leaves & $2.8 \pm 0.3^{\mathrm{C}}$ & $10.8 \pm 0.4^{\mathrm{A}}$ & $3.1 \pm 0.1^{\mathrm{B}}$ & $3.6 \pm 0.1^{\mathrm{AB}}$ & $15.0 \pm 0.8^{\mathrm{B}}$ & $1.0 \pm 0.1^{\mathrm{B}}$ & $76.4 \pm 6.6^{\mathrm{B}}$ & $33.3 \pm 2.3^{\mathrm{AB}}$ & $1.0 \pm 0.3^{\mathrm{B}}$ \\
\hline & MT (Cd) & root & $1.3 \pm 0.1^{\mathrm{A}}$ & $5.0 \pm 0.4^{\mathrm{AB}}$ & $4.0 \pm 0.2^{\mathrm{A}}$ & $3.1 \pm 0.4^{\mathrm{A}}$ & $10.6 \pm 0.4^{\mathrm{AB}}$ & $2.7 \pm 0.4^{\mathrm{A}}$ & $360.9 \pm 29.8^{A}$ & $87.8 \pm 24.5^{\mathrm{A}}$ & $1.9 \pm 0.4^{\mathrm{A}}$ \\
\hline & $\mathrm{Nr}(\mathrm{Cd})$ & leaves & $4.3 \pm 0.3^{\mathrm{AB}}$ & $10.6 \pm 0.4^{\mathrm{A}}$ & $2.7 \pm 0.0^{C}$ & $2.8 \pm 0.2^{C}$ & $16.6 \pm 0.5^{\mathrm{AB}}$ & $1.1 \pm 0.1^{\mathrm{B}}$ & $77.4 \pm 4.6^{\mathrm{B}}$ & $73.3 \pm 31.5^{\mathrm{A}}$ & $1.1 \pm 0.2^{\mathrm{B}}$ \\
\hline & $\mathrm{Nr}(\mathrm{Cd})$ & root & $0.8 \pm 0.4^{\mathrm{A}}$ & $2.7 \pm 1.4^{\mathrm{B}}$ & $1.9 \pm 1.0^{\mathrm{B}}$ & $2.1 \pm 1.1^{\mathrm{A}}$ & $6.8 \pm 3.4^{\mathrm{B}}$ & $2.0 \pm 1.0^{\mathrm{A}}$ & $249.5 \pm 129.4^{\mathrm{A}}$ & $227.6 \pm \pm 195.6^{\mathrm{A}}$ & $1.4 \pm 0.8^{\mathrm{A}}$ \\
\hline & dgt (Cd) & leaves & $3.3 \pm 0.6^{\mathrm{AB}}$ & $9.2 \pm 0.5^{\mathrm{B}}$ & $2.4 \pm 0.1^{\mathrm{D}}$ & $2.7 \pm 0.2^{\mathrm{C}}$ & $16.6 \pm 0.8^{\mathrm{AB}}$ & $1.3 \pm 0.1^{\mathrm{A}}$ & $79.1 \pm 13.3^{\mathrm{B}}$ & $29.7 \pm 2.4^{\mathrm{AB}}$ & $1.5 \pm 0.3^{\mathrm{B}}$ \\
\hline & dgt (Cd) & root & $1.3 \pm 0.1^{\mathrm{A}}$ & $4.7 \pm 0.4^{\mathrm{AB}}$ & $2.9 \pm 0.2^{\mathrm{AB}}$ & $3.0 \pm 0.5^{\mathrm{A}}$ & $12.2 \pm 0.6^{\mathrm{AB}}$ & $3.3 \pm 0.1^{A}$ & $226.2 \pm 18.2^{\mathrm{A}}$ & $58.9 \pm 8.7^{\mathrm{A}}$ & $1.8 \pm 0.4^{\mathrm{A}}$ \\
\hline \multirow[t]{20}{*}{ Grafted plant } & MT & scion & $4.1 \pm 0.1^{\mathrm{ab}}$ & $11.5 \pm 0.1^{\mathrm{ab}}$ & $3.5 \pm 0.1^{\mathrm{ab}}$ & $3.4 \pm 0.1^{\mathrm{ab}}$ & $18.5 \pm 0.3^{\mathrm{bc}}$ & $1.2 \pm 0.0^{\mathrm{b}}$ & $76.5 \pm 7.8^{\mathrm{a}}$ & $38.4 \pm 1.8^{\mathrm{ab}}$ & $2.7 \pm 0.3^{a}$ \\
\hline & MT & rootstock & $1.4 \pm 0.1^{\mathrm{bc}}$ & $7.0 \pm 1.1^{\mathrm{a}}$ & $2.8 \pm 0.6^{\mathrm{b}}$ & $1.7 \pm 0.0^{\mathrm{cd}}$ & $14.0 \pm 0.6^{\mathrm{bc}}$ & $2.3 \pm 0.1^{b}$ & $668.4 \pm 168.7^{\mathrm{ab}}$ & $134.5 \pm 38.9^{b}$ & $8.3 \pm 1.2^{\mathrm{a}}$ \\
\hline & MT & scion & $4.1 \pm 0.1^{\mathrm{ab}}$ & $10.1 \pm 0.6^{\mathrm{ab}}$ & $3.0 \pm 0.1^{\mathrm{ab}}$ & $3.0 \pm 0.2^{\mathrm{bc}}$ & $20.5 \pm 0.6^{\mathrm{ab}}$ & $1.6 \pm 0.1^{\mathrm{a}}$ & $79.4 \pm 9.0^{\mathrm{a}}$ & $36.5 \pm 4.5^{\mathrm{ab}}$ & $2.9 \pm 0.1^{\mathrm{a}}$ \\
\hline & $\mathrm{Nr}$ & rootstock & $2.3 \pm 0.3^{\mathrm{a}}$ & $5.8 \pm 0.3^{\mathrm{ab}}$ & $2.6 \pm 0.1^{\mathrm{b}}$ & $2.1 \pm 0.0^{\mathrm{ab}}$ & $18.7 \pm 0.2^{\mathrm{a}}$ & $3.1 \pm 0.2^{\mathrm{ab}}$ & $379.7 \pm 48.2^{\mathrm{b}}$ & $231.4 \pm 21.2^{\mathrm{a}}$ & $20.0 \pm 1.2^{\mathrm{a}}$ \\
\hline & MT & scion & $4.0 \pm 0.2^{\mathrm{ab}}$ & $12.0 \pm 0.3^{\mathrm{a}}$ & $3.6 \pm 0.3^{a}$ & $3.8 \pm 0.4^{\mathrm{a}}$ & $18.3 \pm 0.5^{\mathrm{cd}}$ & $1.4 \pm 0.1^{\mathrm{ab}}$ & $77.9 \pm 5.9^{\mathrm{a}}$ & $20.4 \pm 1.1^{\mathrm{cd}}$ & $2.1 \pm 0.4^{\mathrm{ab}}$ \\
\hline & dgt & rootstock & $1.4 \pm 0.2^{\mathrm{bc}}$ & $4.7 \pm 0.4^{\mathrm{bc}}$ & $3.8 \pm 0.8^{b}$ & $2.3 \pm 0.3^{\mathrm{ab}}$ & $14.4 \pm 1.7^{\mathrm{bc}}$ & $3.7 \pm 1.1^{\mathrm{a}}$ & $267.8 \pm 36.9^{b}$ & $86.1 \pm 14.3^{b}$ & $3.2 \pm 0.1^{\mathrm{a}}$ \\
\hline & $\mathrm{Nr}$ & scion & $4.6 \pm 0.6^{\mathrm{a}}$ & $9.9 \pm 0.2^{\mathrm{bc}}$ & $2.9 \pm 0.1^{b}$ & $3.0 \pm 0.2^{\mathrm{bc}}$ & $19.8 \pm 0.8^{\mathrm{ab}}$ & $1.3 \pm 0.1^{\mathrm{ab}}$ & $70.7 \pm 5.2^{\mathrm{a}}$ & $44.0 \pm 5.1^{\mathrm{a}}$ & $2.6 \pm 0.2^{\mathrm{a}}$ \\
\hline & $\mathrm{Nr}$ & rootstock & $1.8 \pm 0.4^{\mathrm{ab}}$ & $5.4 \pm 0.4^{\mathrm{ab}}$ & $3.0 \pm 0.4^{\mathrm{b}}$ & $1.9 \pm 0.2^{\mathrm{ab}}$ & $17.0 \pm 0.4^{\mathrm{ab}}$ & $4.6 \pm 2.3^{\mathrm{ab}}$ & $532.9 \pm 146.7^{\mathrm{ab}}$ & $224.6 \pm 72.0^{\mathrm{a}}$ & $60.1 \pm 56.9^{a}$ \\
\hline & dgt & scion & $2.5 \pm 0.6^{\mathrm{b}}$ & $8.9 \pm 1.1^{c}$ & $3.2 \pm 0.4^{\mathrm{ab}}$ & $2.6 \pm 0.3^{c}$ & $15.8 \pm 1.6^{\mathrm{e}}$ & $1.3 \pm 0.0^{\mathrm{b}}$ & $67.4 \pm 5.5^{\mathrm{a}}$ & $17.5 \pm 1.9^{\mathrm{d}}$ & $1.6 \pm 0.2^{\mathrm{b}}$ \\
\hline & dgt & rootstock & $0.8 \pm 0.1^{d}$ & $4.1 \pm 0.6^{\mathrm{bc}}$ & $3.4 \pm 0.3^{\mathrm{b}}$ & $1.3 \pm 0.3^{d}$ & $10.6 \pm 1.4^{\mathrm{d}}$ & $4.4 \pm 2.4^{\mathrm{ab}}$ & $337.5 \pm 6.7^{b}$ & $89.4 \pm 25.4^{b}$ & $3.8 \pm 0.9^{a}$ \\
\hline & MT & scion & $2.8 \pm 1.4^{\mathrm{ab}}$ & $9.8 \pm 0.5^{\mathrm{bc}}$ & $3.3 \pm 0.1^{\mathrm{ab}}$ & $3.8 \pm 0.1^{a}$ & $20.9 \pm 0.3^{\mathrm{ab}}$ & $1.2 \pm 0.1^{\mathrm{b}}$ & $52.5 \pm 22.2^{\mathrm{a}}$ & $30.7 \pm 3.5^{\mathrm{bc}}$ & $2.0 \pm 0.3^{\mathrm{ab}}$ \\
\hline & MT (Cd) & rootstock & $1.1 \pm 0.1^{\mathrm{cd}}$ & $4.4 \pm 0.6^{\mathrm{bc}}$ & $3.0 \pm 0.7^{b}$ & $2.4 \pm 0.2^{\mathrm{a}}$ & $14.3 \pm 0.8^{\mathrm{bc}}$ & $2.1 \pm 0.1^{b}$ & $573.6 \pm 45.3^{\mathrm{ab}}$ & $105.8 \pm 12.9^{b}$ & $3.7 \pm 0.9^{\mathrm{a}}$ \\
\hline & MT & scion & $4.1 \pm 0.7^{\mathrm{ab}}$ & $10.6 \pm 1.0^{\mathrm{ab}}$ & $3.3 \pm 0.3^{\mathrm{ab}}$ & $3.1 \pm 0.2^{\mathrm{bc}}$ & $20.5 \pm 0.7^{\mathrm{ab}}$ & $1.4 \pm 0.1^{\mathrm{ab}}$ & $70.3 \pm 5.2^{\mathrm{a}}$ & $38.5 \pm 6.5^{\mathrm{ab}}$ & $2.5 \pm 0.6^{\mathrm{ab}}$ \\
\hline & $\mathrm{Nr}(\mathrm{Cd})$ & rootstock & $1.8 \pm 0.3^{\mathrm{ab}}$ & $4.8 \pm 0.5^{\mathrm{bc}}$ & $4.4 \pm 0.8^{\mathrm{b}}$ & $2.4 \pm 0.2^{\mathrm{a}}$ & $16.2 \pm 1.4^{\mathrm{ab}}$ & $3.1 \pm 0.6^{\mathrm{ab}}$ & $385.7 \pm 102.1^{b}$ & $103.3 \pm 22.8^{b}$ & $49.6 \pm 34.0^{\mathrm{a}}$ \\
\hline & MT & scion & $4.2 \pm 0.3^{\mathrm{ab}}$ & $10.2 \pm 0.3^{\mathrm{ab}}$ & $3.6 \pm 0.1^{\mathrm{a}}$ & $4.0 \pm 0.2^{\mathrm{a}}$ & $21.4 \pm 0.4^{\mathrm{a}}$ & $0.8 \pm 0.3^{c}$ & $69.8 \pm 3.2^{\mathrm{a}}$ & $23.1 \pm 1.9^{\mathrm{cd}}$ & $2.0 \pm 0.3^{\mathrm{ab}}$ \\
\hline & dgt (Cd) & rootstock & $1.20 .1^{\mathrm{bc}}$ & $3.8 \pm 0.3^{c}$ & $3.8 \pm 0.1^{\mathrm{b}}$ & $2.0 \pm 0.1^{\mathrm{ab}}$ & $11.5 \pm 0.4^{\mathrm{cd}}$ & $3.1 \pm 0.1^{\mathrm{ab}}$ & $835.1 \pm 262.3^{a}$ & $63.4 \pm 0.9^{\mathrm{b}}$ & $2.5 \pm 0.2^{\mathrm{a}}$ \\
\hline & $\mathrm{Nr}$ & scion & $3.3 \pm 0.3^{\mathrm{ab}}$ & $10.2 \pm 0.1^{\mathrm{ab}}$ & $3.2 \pm 0.2^{\mathrm{ab}}$ & $3.0 \pm 0.1^{\mathrm{bc}}$ & $20.7 \pm 0.5^{\mathrm{ab}}$ & $1.1 \pm 0.0^{\mathrm{b}}$ & $61.3 \pm 4.4^{\mathrm{a}}$ & $29.1 \pm 1.0^{\mathrm{bc}}$ & $1.6 \pm 0.1^{b}$ \\
\hline & $\mathrm{Nr}(\mathrm{Cd})$ & rootstock & $1.3 \pm 0.1^{\mathrm{bc}}$ & $5.7 \pm 0.3^{\mathrm{ab}}$ & $16.9 \pm 10.3^{\mathrm{a}}$ & $2.4 \pm 0.2^{\mathrm{a}}$ & $15.0 \pm 1.5^{\mathrm{b}}$ & $3.1 \pm 0.3^{\mathrm{ab}}$ & $505.6 \pm 123.7^{\mathrm{ab}}$ & $50.8 \pm 3.2^{\mathrm{b}}$ & $3.5 \pm 1.3^{\mathrm{a}}$ \\
\hline & dgt & scion & $2.7 \pm 0.3^{\mathrm{ab}}$ & $9.6 \pm 0.4^{\mathrm{bc}}$ & $3.3 \pm 0.2^{\mathrm{ab}}$ & $3.0 \pm 0.1^{\mathrm{bc}}$ & $17.9 \pm 0.8^{\mathrm{de}}$ & $1.3 \pm 0.1^{\mathrm{ab}}$ & $80.1 \pm 9.4^{\mathrm{a}}$ & $20.8 \pm 2.3^{\mathrm{cd}}$ & $1.6 \pm 0.1^{\mathrm{b}}$ \\
\hline & dgt (Cd) & rootstock & $0.9 \pm 0.0^{\mathrm{d}}$ & $4.4 \pm 0.3^{\mathrm{bc}}$ & $4.0 \pm 0.4^{b}$ & $1.8 \pm 0.1^{\mathrm{bc}}$ & $9.4 \pm 0.4^{\mathrm{d}}$ & $2.3 \pm 0.2^{\mathrm{b}}$ & $478.6 \pm 125.1^{\mathrm{ab}}$ & $61.7 \pm 9.2^{\mathrm{b}}$ & $2.4 \pm \pm 0.5^{\mathrm{a}}$ \\
\hline
\end{tabular}




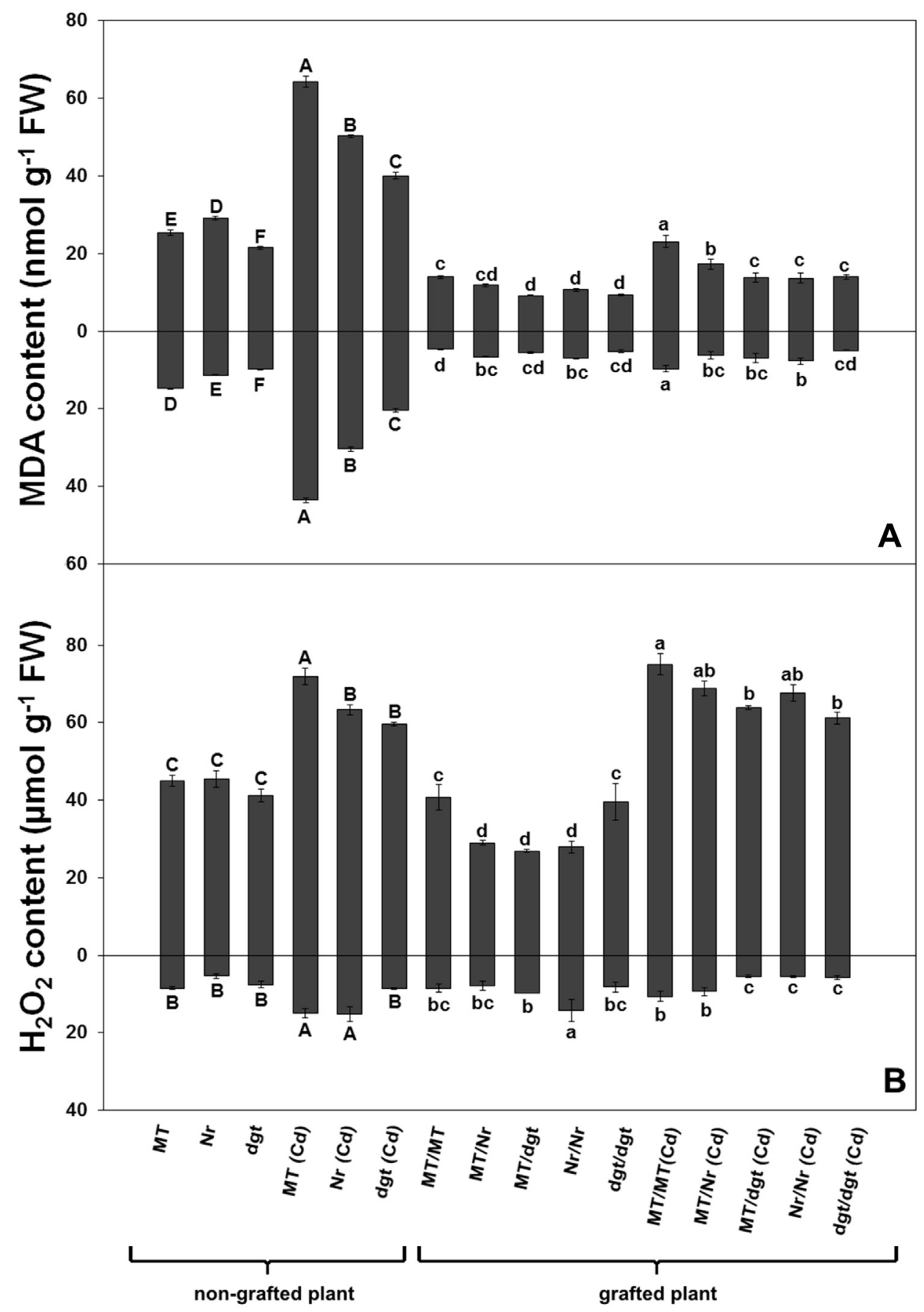

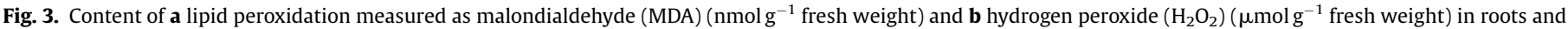

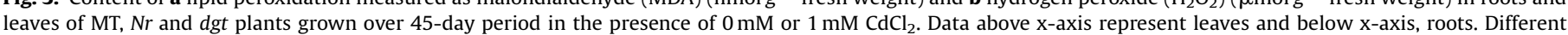
uppercase letters on the top of the columns indicates non-grafted plants and lowercase indicates grafted plants with significantly different at $\mathrm{P}<0.05$ by Duncan test.

\subsection{Lipid peroxidation and $\mathrm{H}_{2} \mathrm{O}_{2}$ content}

Lipid peroxidation, expressed as MDA content, was more pronounced in leaves than in roots for all genotypes of grafted and non-grafted plants (Fig. $3 \mathrm{~A}$ ). In the presence of $\mathrm{Cd}$, non-grafted plants exhibited pronounced increases in the MDA content in the leaves and roots compared with non-grafted control plants (Fig. 3A). The same pattern was observed among the scions of all genotypes of grafted plants following Cd application, which increased lipid peroxidation rates (Fig. 3A); this effect was more pronounced in scions of grafted MT(-Cd)/MT(+Cd) and MT(-Cd)/Nr
(+Cd) plants. The $\mathrm{H}_{2} \mathrm{O}_{2}$ content was higher in leaves of grafted and non-grafted plants exposed to $\mathrm{CdCl}_{2}$ application (Fig. 3B), whereas roots of $d g t$ non-grafted plants appeared to be less affected by $\mathrm{Cd}$ exposure.

\subsection{Chlorophyll content}

The chlorophyll content differed among genotypes and $\mathrm{Cd}$ exposure treatments (Fig. 4). It is clear that the chlorophyll content was less affected by $\mathrm{Cd}$ exposure in dgt plants (Fig. 4). Interesting, the scions of MT plants grafted onto dgt rootstocks exhibited a high 


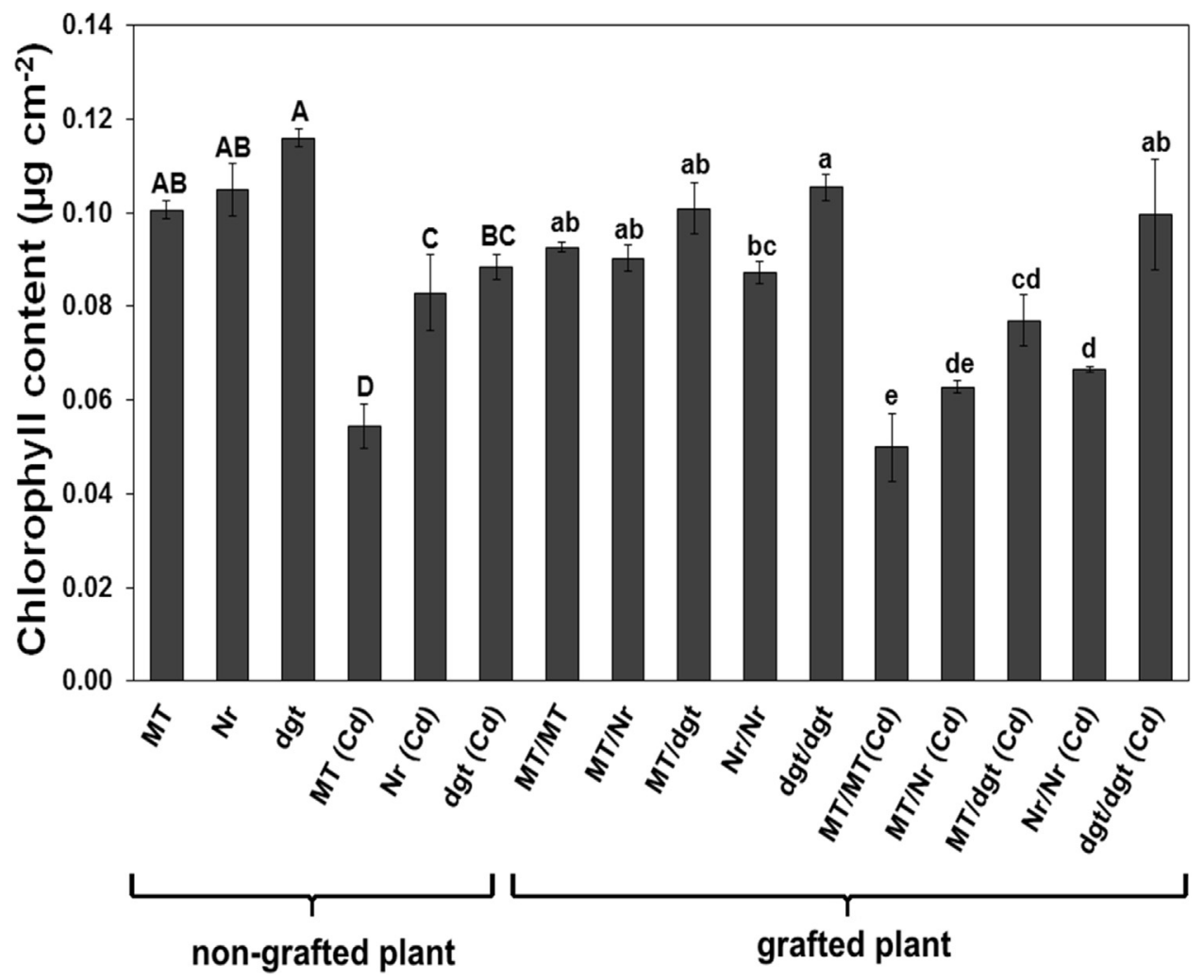

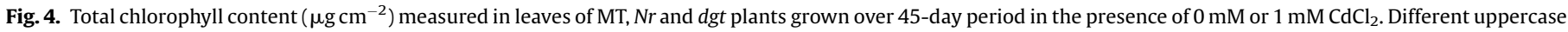
letters on the top of the columns indicates non-grafted plants and lowercase indicates grafted plants with significantly different at $\mathrm{P}<0.05$ by Duncan test.

chlorophyll content compared with scions of MT plants grafted onto either MT or $\mathrm{Nr}$ rootstocks (Fig. 4).

\subsection{Proline content}

Following treatment with $\mathrm{CdCl}_{2}$, non-grafted plants exhibited higher proline contents in both leaves and roots; this effect was more pronounced in $d g t$ mutant plants (Fig. 5). In grafted plants, the proline content was lower in rootstocks subjected to $\mathrm{Cd}$ application. On the other hand, scions of grafted MT(-Cd)/MT(+Cd) and $\mathrm{MT}(-\mathrm{Cd}) / \mathrm{Nr}(+\mathrm{Cd})$ plants exhibited higher proline contents compared with grafted plants without Cd (Fig. 5).

\subsection{Antioxidant enzyme activities}

Three common SOD isoenzymes were detected in leaves (Fig. 6A) and roots (Fig. 6B), which were characterized as Mn/ SOD (SOD I and II) and Cu/Zn-SOD (SOD III) (data not shown). Changes were observed between tissue type and $\mathrm{Cd}$ treatment. For instance, SOD I and II were more pronounced in the roots of both grafted and non-grafted plants (Fig. 6B). Following Cd application, the activity of SOD III decreased to almost zero in the leaves and roots of non-grafted plants (Fig. 6, lanes 4-6), although nongrafted $d g t$ leaves also exhibited very low SOD III activity without Cd (Fig. 6A, lane 3). The activity of SOD I in leaves was affected when $-\mathrm{Cd}$ scions were grafted onto $+\mathrm{Cd}$ rootstocks, independent of the genotype/combination, leading to an increase in SOD I activity (Fig. 6A, lanes 12-16).

CAT (Fig. 7A), APX (Fig. 7B) and GR (Fig. 7C) activities are crucial for the detoxification of any excess $\mathrm{H}_{2} \mathrm{O}_{2}$ produced by SOD and/or by other metabolic processes. Leaves of non-grafted plants exhibited increases in APX, CAT and GR activities following Cd application, whilst scions of grafted plants exposed to Cd exhibited increases in CAT (Fig. 7A) and APX (Fig. 7B) activities. When roots of grafted and non-grafted plants were analysed, an interesting tendency was observed: the application of $\mathrm{Cd}$ triggered differences in enzyme activities among genotypes of non-grafted and grafted plants (Fig. 7). Non-grafted MT plants exposed to Cd application exhibited increased CAT, APX and GR activities, whereas $\mathrm{Nr}$ plants exhibited decreased CAT activity (Fig. 7A). Grafted MT(-Cd)/dgt $(+\mathrm{Cd})$ and $\mathrm{MT}(-\mathrm{Cd}) / \mathrm{Nr}(+\mathrm{Cd})$ plants maintained low APX activity compared with MT(-Cd)/MT(+Cd) plants. On the other hand, MT $(-\mathrm{Cd}) / \mathrm{MT}(+\mathrm{Cd})$ plants exhibited decreased GR activity (Fig. 7B) compared with grafted MT(-Cd)/dgt $(+\mathrm{Cd})$ and $\mathrm{MT}(-\mathrm{Cd}) / \mathrm{Nr}(+\mathrm{Cd})$ plants.

\section{Discussion}

When plants are subjected to environmental stresses, their development can be drastically affected and their growth and yield may be reduced due to changes in metabolism. The stress perception, signal transduction and communication between root and shoot can involve complex mechanisms, including antioxidant system (Gratão et al., 2015), and these mechanisms seem to be modulated by auxin and ethylene (Monteiro et al., 2011, 2012; Gratão et al., 2012). We used hormonal mutants and the grafting technique to highlight the role of auxin and ethylene in stress (Cd exposure) perception in roots and signalling to the upper plant parts. Cd toxicity has obvious negative effects on plant growth (Fidalgo et al., 2011; Gallego et al., 2012; Anjum et al., 2015) and can inhibit root dry weight, root diameter and number of lateral roots (Shafi et al., 2010). Inhibition of $40 \%$ in root growth was clearly observed in MT non-grafted plants exposed to $\mathrm{CdCl}_{2}$ (Fig. 2). Among grafted plants, a pronounced decrease in the growth of both stressed and non-stressed plants was also observed (Fig. 2). During graft formation, plants undergo numerous processes, 


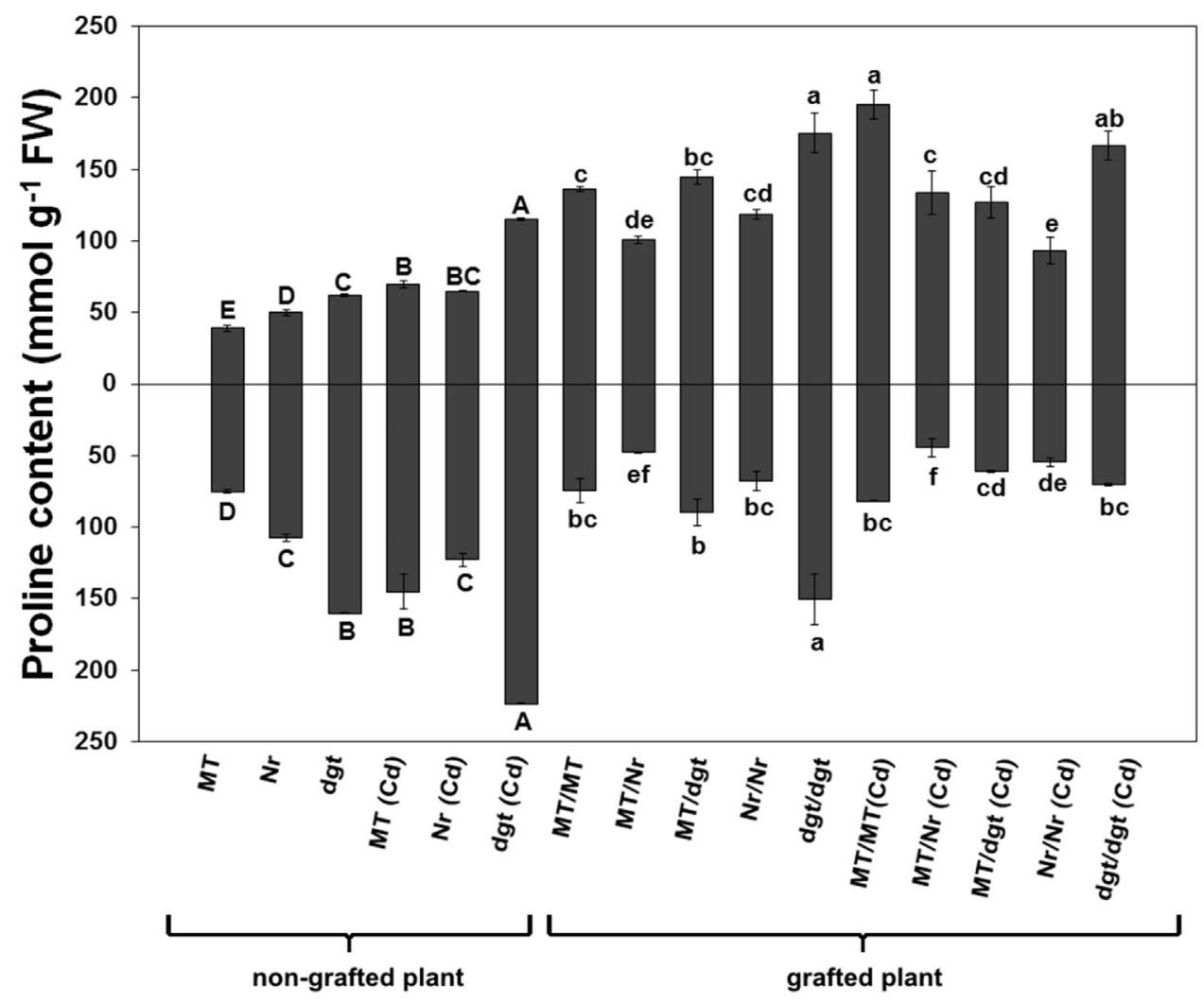

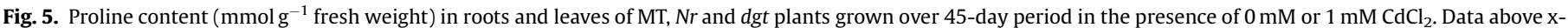

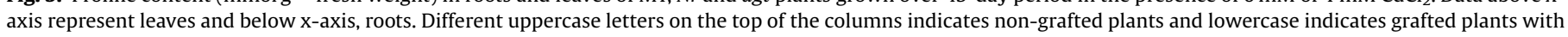
significantly different at $\mathrm{P}<0.05$ by Duncan test.

including adhesion of the rootstock and scion, cell proliferation, connection of the vascular tissues and then resumption of growth (Melnyk et al., 2015). This might be the main reason for the low biomass accumulation and might also explain the results obtained. Furthermore, the biomass accumulation was clearly independent of genotype or Cd treatment.
Roots are the first organs to come into contact with $\mathrm{Cd}$. This tissue has been shown to accumulate high concentrations of $\mathrm{Cd}$ in the majority of plant species (Pereira et al., 2002; Gallego et al., 2012). The data obtained in this work confirmed previous reports of high Cd accumulation in roots and rootstocks, with the Cd content varying considerably among genotypes (Table 1). Cd
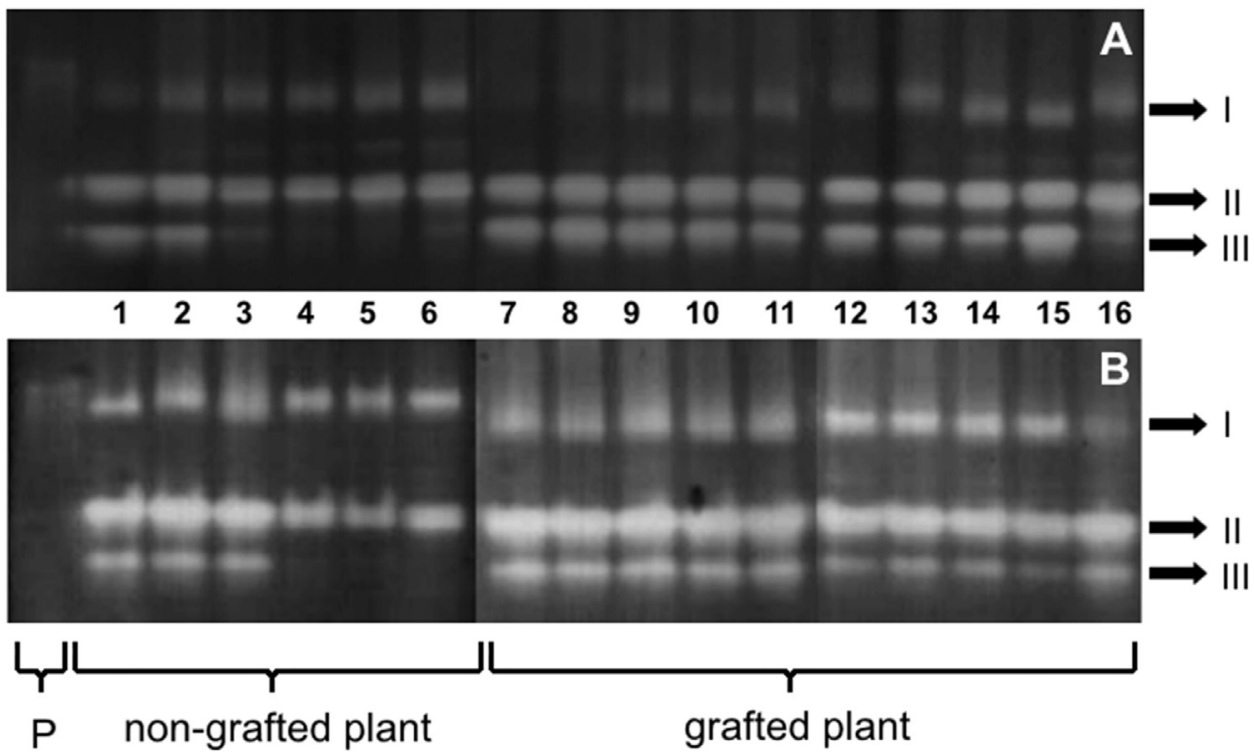

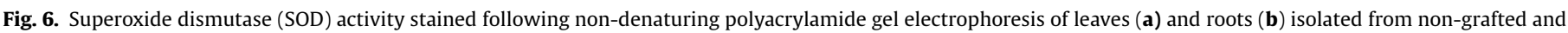

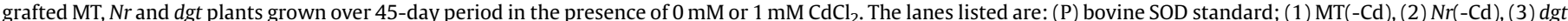

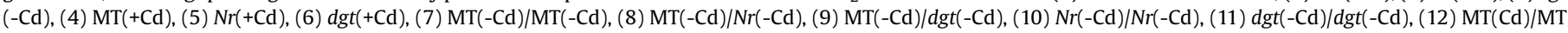
(+Cd), (13) MT(-Cd)/Nr(+Cd), (14) MT(-Cd)/dgt(+Cd), (15) $N r(-\mathrm{Cd}) / N r(+\mathrm{Cd})$ and (16) $d g t(-\mathrm{Cd}) / \operatorname{dgt}(+\mathrm{Cd})$.The SOD isoforms are (I) Mn-SOD, (II) Mn-SOD; (III) Cu/Zn-SOD. 


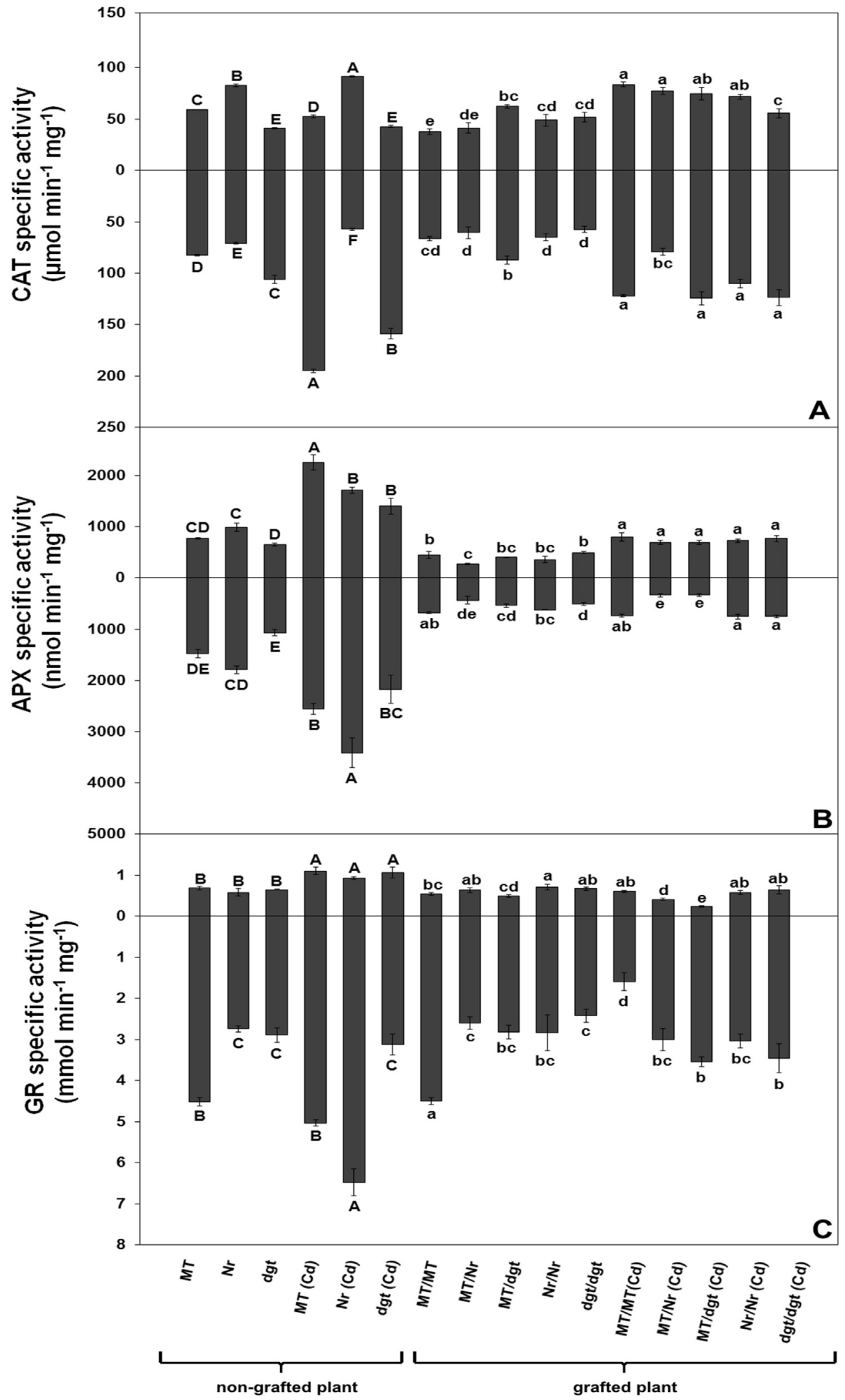

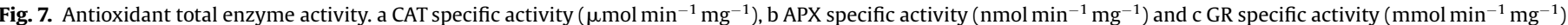

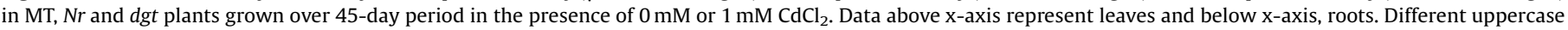
letters on the top of the columns indicates non-grafted plants and lowercase indicates grafted plants with significantly different at $\mathrm{P}<0.05$ by Duncan test. 
perception and translocation trigger signal transduction cascades and can be associated with hormones (Asgher et al., 2015). In a previous ultrastructural study using MT tomato plants and hormonal mutants subjected to Cd exposure (Gratão et al., 2009), the stomata of MT plants were closed, whereas the majority of stomata of $\mathrm{Nr}$ and $d g t$ mutants remained open under Cd stress conditions. This might suggest that $\mathrm{Cd}$ accumulation was higher in non-grafted mutants due to transpiration, based on the open stomata. Moreover, $\mathrm{Nr}$ plants accumulate more $\mathrm{Cd}$ in leaves when compared with MT and dgt plants, which can suggest that ethylene can down-regulate this mechanism, because $N r$ plants exhibit better ability of $\mathrm{Cd}$ translocation via xylem, once it is the main physiological process determining the Cd accumulation in shoots (Uraguchi et al., 2009). Cd accumulation in rootstocks of grafted plants subjected to Cd showed a distinct trend compared with nongrafted plants (Table 1). The rootstocks of grafted hormonal mutants exhibited decreases in $\mathrm{Cd}$ accumulation compared with MT. The rootstocks were exposed to $\mathrm{Cd}$ at the time of grafting, which suggests that inhibition of auxin and ethylene perception may have contributed to the increase in Cd over time.

Since $\mathrm{Cd}$ can induce plant structural and biochemical barriers that can control the loading and unloading of elements (Gratão et al., 2009; Lux et al., 2011). Certainly, these responses are controlled by hormones, such as auxin and ethylene. In fact, Gratão et al. (2009) observed that Cd induced distinct ultrastructural patterns in roots of MT and hormonal mutants, such as root diameter, disintegration of the epidermis and the external layers of the cortex. These alterations can result in differences in the nutritional status of the genotypes (Table 2). For instance, rootstocks of grafted $\mathrm{MT}(-\mathrm{Cd}) / \mathrm{Nr}(+\mathrm{Cd})$ and $\mathrm{Nr}(-\mathrm{Cd}) / \mathrm{Nr}(+\mathrm{Cd})$ plants exhibited an intense decrease in Mn concentration (66\% and $77 \%$, respectively) compared with the grafted control. In the presence of $\mathrm{Cd}$, the uptake of polyvalent cations, such as $\mathrm{Mn}$, can decrease due to Cd interaction with essential nutrients (Gonçalves et al., 2009). Self-grafting combinations with $\mathrm{Cd}$ application exhibited increases in the $S$ concentration of rootstocks of self-grafted MT/MT and dgt/ dgt plants compared with the same combination without $\mathrm{Cd}$. It is well known that $\mathrm{Cd}$ may be detoxified in plants through the chelation of metal ions with high-affinity phytochelatins, a family of S-rich peptides, which indicates the association of S-containing groups as an important defence response against Cd toxicity (Bashir et al., 2015). Moreover, scions of grafted MT(-Cd)/MT(+Cd) plants increase $20 \% \mathrm{~S}$ concentrations compared with grafted MT $(-\mathrm{Cd}) / \mathrm{Nr}(+\mathrm{Cd})$ plants, suggesting that different rootstocks may exhibit dissimilar abilities in mineral uptake efficiencies. The data suggest that the uptake and translocation of S during Cd stress may be associated with ethylene signalling, considering that interplay between ethylene signalling pathway and the transduction of the signal of S deficit (Moniuszko, 2015). Yet, it would be particularly interesting in future research to investigate phytochelatins synthesis and action in the different genotypes of tomato used and under the same grafting combinations used in this study.

On the other hand, rootstocks of grafted MT(-Cd)/dgt $(+C d)$ plants showed an increased Fe content, whereas the Mg content increased in the rootstocks of grafted $\mathrm{Nr}(-\mathrm{Cd}) / \mathrm{Nr}(+\mathrm{Cd})$ plants. These elements play a role in preventing oxidative stress binding of proteins and enzymes (Hänsch and Mendel, 2009), which can contribute to the alleviation of $\mathrm{Cd}$ stress. Among all treatments, the dgt plants appeared to take up and translocate less macro- and micronutrients compared with $\mathrm{Nr}$ and MT. Auxin synthesis and transport contribute to the establishment of auxin gradients for the initiation of lateral roots in the pericycle (Fukaki and Tasaka, 2009). The dgt tomato mutant is relatively insensitive to auxin, which leads to less lateral root formation as well as dry mass reduction (Fig. 2), which in turn may result in are reduction in nutrient uptake and Cd accumulation because non-grafted $d g t$ and $d g t(-C d) /$
$\operatorname{dgt}(+\mathrm{Cd})$ plants accumulated $17.7 \%$ and $55.8 \%$ less $\mathrm{Cd}$ in roots compared with $\mathrm{Nr}$ and $\mathrm{Nr}(-\mathrm{Cd}) / \mathrm{Nr}(+\mathrm{Cd})$ plants (Table 1). Although ethylene induces modifications of auxin synthesis and transport (Fukaki and Tasaka, 2009), the genotype $\mathrm{Nr}$ did not exhibit any major changes in nutrient uptake and root dry mass as exhibited by the dgt plants. Although the dgt plants exhibited low nutrient contents, increases in the Fe content in the leaves of non-grafted plants and the rootstocks of grafted MT(-Cd)/dgt $(+\mathrm{Cd})$ plants were observed.

A large number of studies indicated that auxin and ethylene can modulate oxidative stress (Djanaguiraman et al., 2011; Krishnamurthy and Rathinasabapathi, 2013). However, we observed that non-grafted dgt and $\mathrm{Nr}$ plants appeared to be less affected by $\mathrm{Cd}$ stress conditions because they exhibited the lowest MDA and $\mathrm{H}_{2} \mathrm{O}_{2}$ contents in both tissue types (Fig. 3A and B), even though more Cd was accumulated compared with the MT genotype (Table 1). Therefore, auxin and ethylene suppression led to a smaller effect on cell homeostasis because lipid peroxidation may trigger membrane leakage (Talukdar, 2011; Piwowarczyk et al., 2016). Our results are in accordance with other studies, where the inhibition of auxin and ethylene perception can minimize Cd toxic effects through increased membrane stability (Iakimova et al., 2008; Monteiro et al., 2011).

Grafted plants whose rootstocks originated from plants exposed to $\mathrm{CdCl}_{2}$ exhibited higher lipid peroxidation rates and $\mathrm{H}_{2} \mathrm{O}_{2}$ accumulation in the scions (Fig. $3 \mathrm{~A}$ and $\mathrm{B}$ ), although low $\mathrm{Cd}$ concentrations were observed (Table 1 ), suggesting that the stress caused by $\mathrm{Cd}$ in the rootstock was signalled to the scion. It is interesting to note that grafted $\mathrm{MT}(-\mathrm{Cd}) / \mathrm{MT}(+\mathrm{Cd})$ plants exhibited high lipid peroxidation, which indicates that low sensitivity to auxin and ethylene can regulate developmental responses under environmental stress. Moreover, MT scions exhibited changes in MDA content depending on the rootstock combination. For instance, rootstocks of grafted $\mathrm{Nr}(+\mathrm{Cd})$ and $d g t(+\mathrm{Cd})$ plants triggered different responses in MT scions compared with MT $(-\mathrm{Cd}) / \mathrm{MT}(+\mathrm{Cd})$ plants, minimizing lipid peroxidation expressed as MDA content in $25.3 \%$ and $40.2 \%$, respectively (Fig. 3A).

Membrane selectivity and mechanisms of heavy metal immobilization can prevent $\mathrm{Cd}$ uptake and translocation to the chloroplast (Siedlecka and Krupa, 1999). Nonetheless, Cd can circumvent these barriers and accumulate in chloroplasts, which exhibit changes in organelle shape and internal organization (Gratão et al., 2009), reducing their content per cell and per unit leaf area (Fagioni et al., 2009). Moreover, Cd toxicity can reduce the chlorophyll content of several plant species (Lysenko et al., 2015; Muradoglu et al., 2015). These alterations in the chloroplasts of plants exposed to Cd may occur due to an increase in ROS production in the leaves (Fig. 3B). In our study, the chlorophyll contents were lower in grafted and non-grafted plants subjected to $\mathrm{CdCl}_{2}$ application (Fig. 4). The chlorophyll content in non-grafted MT plants was more strongly affected by $\mathrm{Cd}$ compared with hormonal mutant plants. This result may be explained by the high lipid peroxidation of the chloroplast membrane of MT plants measured as the MDA content (Fig. 3A).

In contrast, the chlorophyll content decreased $35 \%$ in scions of grafted MT(-Cd)/MT(+Cd) plants compared with $\mathrm{MT}(-\mathrm{Cd}) / \operatorname{dgt}(+\mathrm{Cd})$ and $20.6 \%$ compared with $\mathrm{MT}(-\mathrm{Cd}) / \mathrm{Nr}(+\mathrm{Cd})$ plants (Fig. 4), indicating possible differences as to how mutants and MT rootstocks may transmit information to MT scions. It is interesting to note that dgt plants exhibited a high chlorophyll content compared with the MT and $\mathrm{Nr}$ genotypes, as verified in grafted and non-grafted plants subjected to the stress condition. These results can be associated with the size and number of starch grains in the dgt genotype, which were not reduced, in contrast to the MT and $\mathrm{Nr}$ starch grains, when subjected to Cd application (Gratão et al., 2009). On the other hand, it is interesting to note that the dgt plants 
appeared to exhibit leaves with a dark green phenotype due to a reduction in cell size, which causes an increased concentration of chloroplasts per leaf area (Koornneef et al., 1990) and can result in high chlorophyll content, but this requires further elucidation under stress conditions. A more detailed study focused exclusively on ultrastructural changes in grafted and non-grafted plant models is currently underway in our laboratory.

As a direct response to stress, plants enhance the synthesis and reduce the degradation of protective metabolites such as proline, which contribute to stabilization of protein molecules and membranes (Seregin and Kozhevnikova, 2006). High proline contents in both tissue types of non-grafted plants exposed to $\mathrm{Cd}$, particularly the dgt plants, were observed (Fig. 5). Although proline can be associated with ROS scavenging (Zouari et al., 2016b), the $\mathrm{H}_{2} \mathrm{O}_{2}$ content was high in plants exposed to Cd (Fig. 3B), indicating there was insufficient proline to control the oxidation. Scions of grafted MT(-Cd)/MT(+Cd) plants exhibited $31.2 \%$ more proline content compared with scions of grafted MT(-Cd)/ $\mathrm{Nr}(+\mathrm{Cd})$ and of $35 \%$ in $\mathrm{MT}(-\mathrm{Cd}) / \operatorname{dg} t(+\mathrm{Cd})$ plants, which suggests that the suppression of auxin and ethylene from the rootstock can regulate proline accumulation in the scion understress conditions.

As a response to $\mathrm{Cd}$ stress conditions, antioxidant enzymes allow plants to avoid or tolerate oxidative stress and to survive environmental adversities (Gallego et al., 2012; Gratão et al., 2015). The antioxidant enzymes SOD, APX, CAT and GR were selected based on several reports on their responses to Cd stress in plants. These enzymes exhibited varied responses among genotypes, grafting combinations, tissue types and $\mathrm{Cd}$ treatment.

SOD, which converts $\mathrm{O}_{2}{ }^{-}-$into $\mathrm{H}_{2} \mathrm{O}_{2}$, has been shown to be induced in a number of plant species when exposed to $\mathrm{Cd}$ (Cho and Seo, 2005; Ekmekci et al., 2008; Kapoor et al., 2014). Different isoenzymes contribute to cell protection against different toxic substances, such as Cd (Aksmann et al., 2014). In this study, three SOD isoenzymes (SOD I, II and III) were identified following nondenaturing PAGE analysis (Fig. 6), with a similar isoenzyme classification as previously reported by Gratão et al. (2012, 2015) for tomato. Mn/SOD (SOD I and II) and Cu-Zn/SOD (SOD III) were highly active in both tissue types and were more pronounced in the roots. The leaves of grafted plants exhibited an increase in SOD I activity following Cd application (Fig. 6, lanes 12-16). Cd exposure can induce up-regulation of Mn-SOD at both transcript and activity levels (Rodríguez-Serrano et al., 2006), which supports a slight increase in Mn-SOD activity under Cd stress conditions.

A lower SOD III activity in the roots and leaves of MT, $\mathrm{Nr}$ and $d g t$ non-grafted plants was observed in response to Cd application (Fig. 6, lanes 4-6). Moreover, MT and Nr plants exhibited decreases in $\mathrm{Zn}$ and $\mathrm{Cu}$ concentrations in leaves (Table 2), which might be related to the fact that $\mathrm{Cu}$ and $\mathrm{Zn}$ are cofactors of SOD III (Gill et al., 2015). Although no changes in $\mathrm{Cu}$ and $\mathrm{Zn}$ concentrations occurred in grafted plants, we observed a reduction in SOD III activity following Cd application (Fig. 6, lanes 12-16) because Cd can affect the expression and regulation of this isoenzyme (Romero-Puertas et al., 2007).

$\mathrm{H}_{2} \mathrm{O}_{2}$ produced in response to SOD activity or other metabolic activities can subsequently be reduced to $\mathrm{H}_{2} \mathrm{O}$ by the action of CAT, APX and other peroxidases. Although low concentrations of $\mathrm{Cd}$ accumulated in the scions (Table 1), we observed increases in CAT and APX activities compared with the scions from plants that were not exposed to $\mathrm{Cd}$, indicating stress signalling from the rootstocks to the scions of grafted plants under Cd stress (Fig. 7A and B). CAT activity was lower in the scions of grafted $\operatorname{dgt}(-\mathrm{Cd}) / \operatorname{dg} t(+\mathrm{Cd})$ plants compared with other genotypes, being 33.5\% lower in MT(-Cd)/MT $(+\mathrm{Cd})$. Thus, auxin suppression appears to decrease the scavenging of $\mathrm{H}_{2} \mathrm{O}_{2}$ by CAT, but $d g t$ plants might exhibit other auxinindependent systems to remove $\mathrm{H}_{2} \mathrm{O}_{2}$ because $d g t$ plants exhibited low levels of $\mathrm{H}_{2} \mathrm{O}_{2}$ in scions (Fig. 3B). The up-regulation of APX can increase the ability to overcome Cd stress (Wu et al., 2015). Although APX activity increased in the scions of grafted plants under Cd stress, which indicates signalling of stress from the rootstock to the upper parts of the plant, this might not be mediated by hormones because scions exhibited the same APX activity responses independent of rootstock genotype.

With respect to other antioxidant enzymes, GR plays an important role in maintaining the metabolic balance between GSH and ascorbate (AsA) contents and $\mathrm{H}_{2} \mathrm{O}_{2}$ degradation, which can be involved in heavy metal detoxification (Sharma and Dietz, 2009). The $\mathrm{Nr}$ mutant accumulated more Cd in non-grafted plants (Table 1), which probably induced increases in APX and GR activities (Fig. 7B and C). GR activity requires GSH, an S containing metabolite. Although Cd stress can cause some $S$ deficient, it can be less intense in $\mathrm{Nr}$ plants due to the suppression in ethylene perception, which avoid S deficiency and consequently having little or no effect on GR activity (Moniuszko, 2015). In MT and dgt non-grafted plants exposed to $\mathrm{Cd}$, the leaves did not exhibit changes in GR activity, indicating that stress perception and signalling from the rootstock were inefficient to induce a GR response. A study conducted by Jozefczak et al. (2014), who worked with Arabidopsis, indicated a delayed antioxidant response in leaves as a consequence of $\mathrm{Cd}$ accumulation in the roots. On the other hand, the rootstocks of grafted MT(-Cd)/MT(+Cd) plants exhibited lower GR activity compared with other grafted plants exposed to $\mathrm{Cd}$, being up to $54.9 \%$ lower than $\mathrm{MT}(-\mathrm{Cd}) / \operatorname{dgt}(+\mathrm{Cd})$ plants. Such a result suggests that auxin and ethylene can trigger a signal to regulate GR activity under Cd stress conditions. Such findings suggest the possible interaction of stress signalling from the root to the shoot mediated by auxin and ethylene. This may be related to the subsequent activation of antioxidant defences in scions, observed as an increase in enzyme activity in this tissue (Fig. 7A, B and C).

Plant roots can perceive the environmental condition and propagate the information into a refined network of signals transmitted to the shoot (Shabala et al., 2016). The use of grafted tomato plants revealed distinct trends in crosstalk between antioxidant responses and signalling of plant organs during stress (root-to-shoot) that clearly indicated signalling responses from the rootstocks, allowing sufficient time to activate defence mechanisms in the shoot (Gratão et al., 2015). Moreover, plant hormones are important endogenous factors, and may have an important role in root-to-shoot communication. For instance, Omid et al. (2007) have identified, in the phloem of melon (Cucumis melo L.), transcripts which were associated with signal transduction and part of them, were associated with auxin signalling.

We observed that MT scions exhibited different responses when a hormonal mutant was used as rootstock. However, dgt mutant might avoid better the stress imposed by $\mathrm{Cd}$ than $\mathrm{Nr}$ mutant, because the auxin suppression may decline ethylene production, once it is well known that auxin-stimulated ethylene biosynthesis (Gratão et al., 2009, 2012; Monteiro et al., 2011). Thus, auxin and ethylene plays fundamental communication chemical modes to optimize the performance of shoot antioxidant system during $\mathrm{Cd}$ stress condition in roots

\section{Conclusion}

The results clearly indicated distinct trends among the combinations tested, which indicated that signalling responses from the rootstock to the scion are related to auxin and ethylene, allowing sufficient time for defence mechanisms to be stimulated in the upper parts of the plant in response to the oxidative stress condition induced by the $\mathrm{Cd}$ taken up by the roots. We hoped to obtain more information on root-to-shoot stress modulation using grafting, paying special attention to the responses of the 
antioxidant systems and the role of plant hormones. Such responses based on the interactions between hormones and redox signalling pathways in the control of growth and cross-tolerance to stress can be used to manipulate heavy metal tolerance since auxin and ethylene are directly involved in this mechanism.

\section{Acknowledgements}

This work was funded by Fundação de Amparo à Pesquisa do Estado de São Paulo (FAPESP - Grant no 2013/27080-4). We thank Coordenação de Aperfeiçoamento de Pessoal de Nível Superior (CAPES) (L.R.A) for scholarship granted. We also thank the Conselho Nacional de Desenvolvimento Científico e Tecnológico (CNPq-Brazil) for the research fellowship to R.A.A.

\section{References}

Ahmad, P., Abdel Latef, A.A., Abd Allah, E.F., Hashem, A., Sarwat, M., Anjum, N.A. Gucel, S., 2016. Calcium and potassium supplementation enhanced growth, osmolyte secondary metabolite production, and enzymatic antioxidant machinery in cadmium-Exposed chickpea (Cicer arietinum L.). Front. Plant Sci. 7. 513. doi:http://dx.doi.org/10.3389/fpls.2016.00513.

Aksmann, A., Pokora, W., Baścik-Remisiewicz, A., Dettlaff-Pokora, A., Wielgomas, B. Dziadziuszko, M., Tukaj, Z., 2014. Time-dependent changes in antioxidative enzyme expression and photosynthetic activity of Chlamydomonas reinhardtii cells under acute exposure to cadmium and anthracene. Ecotoxicol. Environ. Saf. 110, 31-40. doi:http://dx.doi.org/10.1016/j.ecoenv.2014.08.005.

Alexieva, V., Sergiev, I., Mapelli, S., Karanov, E., 2001. The effect of drought and ultraviolet radiation on growth and stress markers in pea and wheat. Plant Cell Environ. 24, 1337-1344. doi:http://dx.doi.org/10.1046/j. 13653040.2001.00778.x

Anjum, S.A., Tanveer, M., Hussain, S., Bao, M., Wang, L., Khan, I., Ullah, E., Tung, S.A., Samad, R.A., Shahzad, B., 2015. Cadmium toxicity in Maize (Zea mays L.): consequences on antioxidative systems, reactive oxygen species and cadmium accumulation. Environ. Sci. Pollut. Res. 22, 17022-17030. doi:http://dx.doi.org 10.1007/s11356-015-4882-z.

Asgher, M., Khan, M.I.R., Anjum, N.A., Khan, N.A., 2015. Minimising toxicity of cadmium in plants - role of plant growth regulators. Protoplasma 252, 399-413. doi:http://dx.doi.org/10.1007/s00709-014-0710-4

Azevedo, R.A., Alas, R.M., Smith, R.J., Lea, P.J., 1998. Responses of antioxidant enzymes to transfer from elevated carbon dioxide to air and ozone fumigation, in the leaves and roots of wild-type and catalase-deficient mutant of barley. Physiol. Plant. 104, 280-292. doi:http://dx.doi.org/10.1034/j. 1399_ 3054.1998.1040217.x.

Bankaji, I., Sleimi, N., López-Climent, M.F., Perez-Clemente, R.M., Gomez-Cadenas, A. 2014. Effects of combined abiotic stresses on growth, trace element accumulation, and phytohormone regulation in two halophytic species. J. Plant Growth Regul. 33, 632-643. doi:http://dx.doi.org/10.1007/s00344-014-9413-5.

Bashir, H., Ibrahim, M.M., Bagheril, R., Ahamad, J., Arif, I.A., Baigl, M.A., Qureshi, M.I. 2015. Influence of sulfur and cadmium on antioxidants, phytochelatins and growth in Indian mustard. AoB Plants doi:http://dx.doi.org/10.1093/aobpla/ plv001.

Bates, L.S., Waldren, R.P., Teare, I.D., 1973. Rapid determination of free proline for water-stress studies. Plant Soil 39, 205-207. doi:http://dx.doi.org/10.1007/ BF00018060.

Boaretto, L.F., Carvalho, G., Borgo, L., Creste, S., Landell, M.G.A., Mazzafera, P., Azevedo, R.A., 2014. Water stress reveals differential antioxidant responses of tolerant and non-tolerant sugarcane genotypes. Plant Physiol. Biochem. 74, 165-175. doi:http://dx.doi.org/10.1016/j.plaphy.2013.11.016.

Bradford, M.M.A., 1976. Rapid and sensitive method for the quantitation of microgram quantities of protein utilizing the principle of protein-dye binding. Anal. Biochem. 72, 248-254. doi:http://dx.doi.org/10.1016/0003-2697(76) 90527-3.

Bueso, E., Alejandro, S., Carbonell, P., Perez-Amador, M.A., Fayos, J., Bellés, J.M., 2007. The lithium tolerance of the Arabidopsis cat2 mutant reveals a cross-talk between oxidative stress and ethylene. Plant J. 52,1052-1065. doi:http://dx.doi. org/10.1111/j.1365-313X.2007.03305.X.

Carvalho, R.F., Monteiro, C.C., Caetano, A.C., Dourado, M.N., Gratão, P.L., Haddad, C.K. B., Peres, L.E.P., Azevedo, R.A., 2013. Leaf senescense in tomato mutants as affected by irradiance and phytohormones. Biol. Plant. 57, 749-757. doi:http:// dx.doi.org/10.1007/s10535-013-0333-1.

Castagna, A., Ederli, L., Pasqualini, S., Mensuali-Sodi, A., Baldan, B., Donnini, S., Ranieri, A., 2007. The tomato ethylene receptor LE-ETR3 (NR) is not involved in mediating ozone sensitivity: causal relationships among ethylene emission, oxidative burst and tissue damage. New Phytolo. 174, 342-356.

Chilimba, A.D.C., Young, S.D., Black, C.R., Rogerson, K.B., Ander, E.L., Watts, M., Lammel, J., Broadley, M.R., 2011. Maize grain and soil surveys reveal suboptimal dietary selenium intake is widespread in Malawi. Sci. Rep. doi:http://dx.doi.org $10.1038 /$ srep00072.
Chmielowska-Bak, J., Gzyl, J., Rucinska-Sobkowiak, R., Arasimowicz-Jelonek, M., Deckert, J., 2014. The new insights into cadmium sensing. Front. Plant Sci. 5, 113. doi:http://dx.doi.org/10.3389/fpls.2014.00245.

Cho, U., Seo, N., 2005. Oxidative stress in Arabidopsis thaliana exposed to cadmium is due to hydrogen peroxide accumulation. Plant Sci. 168, 113-120. doi:http://dx. doi.org/10.1016/j.plantsci.2004.07.021.

Djanaguiraman, M., Sheeba, J.A., Durga, D.D., Bangarusamy, U., 2009. Cotton leaf senescence can be delayed by nitrophenolate spray through enhanced antioxidant defence system. J. Agron. Crop Sci. 195, 213-224. doi:http://dx.doi. org/10.1111/j.1439-037X.2009.00360.x.

Djanaguiraman, M., Prasad, P.V.V., Al-Khatib, K., 2011. Ethylene perception inhibitor 1-MCP decreases oxidative damage of leaves through enhanced antioxidant defence mechanisms in soybean plants grown under high temperature stress. Environ. Exp. Bot. 71, 215-223. doi:http://dx.doi.org/10.1016/j.envexpbot. 2010.12.006.

Ekmekci, Y., Tanyolac, D., Ayhana, B., 2008. Effects of cadmium on antioxidant enzyme and photosynthetic activities in leaves of two maize cultivars. J. Plant Physiol. 165, 600-611. doi:http://dx.doi.org/10.1016/j.jplph.2007.01.017.

Fagioni, M., D’Amici, G.M., Timperio, A.M., Zolla, L., 2009. Proteomic analysis of multiprotein complexes in the thylakoid membrane upon cadmium treatment. J. Proteome Res. 8, 310-326. doi:http://dx.doi.org/10.1021/pr800507x.

Fassler, E., Evangelou, M.W., Robinson, B.H., Schulin, R., 2010. Effects of indole-3acetic acid (IAA) on sunflower growth and heavy metal uptake in combination with ethylene diamine disuccini acid (EDDS). Chemosphere 80 doi:http://dx. doi.org/10.1016/j. chemosphere (2010.04.077).

Ferraz, P., Fidalgo, F., Almeida, A., Teixeira, J., 2012. Phytostabilization of nickel by the zinc and cadmium hyperaccumulator Solanumnigrum L. are metallothioneins involved? Plant Physiol. Biochem. 57, 254-260. doi:http://dx.doi.org/10.1016/j. plaphy.2012.05.025.

Fidalgo, F., Freitas, R., Ferreira, R., Pessoa, A.M., Teixeira, J., 2011. Solanum nigrum L. antioxidant defence system isoenzymes are regulated transcriptionally and post translationally in Cd-induced stress. Environ. Exp. Bot. 72, 312-319. doi: http://dx.doi.org/10.1016/j.envexpbot.2011.04.007.

Fukaki, H., Tasaka, M., 2009. Hormone interactions during lateral root formation. Plant Mol. Biol. 69, 437-449. doi:http://dx.doi.org/10.1007/s11103-008-9417-2.

Gallego, S.M., Pena, L.B., Barcia, R.A., Azpilicueta, C.E., Iannone, M.F., Rosales, E.P., Zawoznik, M.S., Groppa, M.D., Benavides, M.P., 2012. Unravelling cadmium toxicity and tolerance in plants: insight into regulatory mechanisms. Environ. Exp. Bot. 86, 33-46. doi:http://dx.doi.org/10.1016/j.envexpbot.2012.04.006.

George, S., Venkataraman, G., Parida, A., 2010. A chloroplast-localized and auxininduced glutathione S-transferase from phreatophyte Prosopisjuli flora confer drought tolerance on tobacco. J. Plant Physiol. 167, 311-318. doi:http://dx.doi. org/10.1016/j.jplph.2009.09.004

Gill1, S.S., Anjum, N.A., Gill, R., Yadav, S., Hasanuzzaman, M., Fujita, M., Mishra, P., Sabat, S.C., Tuteja, N., 2015. Superoxide dismutase-mentor of abiotic stress tolerance in crop plants. Environ. Sci. Pollut. Res. 14, 10375-10394. doi:http:// dx.doi.org/10.1007/s11356-015-4532-5.

Gonçalves, J.F., Antes, F.G., Maldaner, J., Pereira, L.B., Tabaldi, L.A., Rauber, R., Rossato, L.V., Bisognin, D.A., Dressler, V.L., Flores, E.M.M., 2009. Cadmium and mineral nutrient accumulation in potato plantlets grown under cadmium stress in two different experimental culture conditions. Plant Physiol. Biochem. 47, 814-821. doi:http://dx.doi.org/10.1016/j.plaphy.2009.04.002.

Gratão, P.L., Monteiro, C.C., Rossi, M.L., Martinelli, A.P., Peres, L.E.P., Medici, L.O., Lea, P.J., Azevedo, R.A., 2009. Differential ultrastructural changes in tomato hormonal mutants exposed to cadmium. Environ. Exp. Bot. 67, 387-394. doi: http://dx.doi.org/10.1016/j.envexpbot.2009.06.017.

Gratão, P.L., Monteiro, C.C., Carvalho, R.F., Tezotto, T. Piotto, F.A., Peres, L.E.P., Azevedo, R.A., 2012. Biochemical dissection of diageotropica and Never ripe tomato mutants to Cd-stressful conditions. Plant Physiol. Biochem. 56, 79-96. doi:http://dx.doi.org/10.1016/j.plaphy.2012.04.009.

Gratão, P.L., Monteiro, C.C., Tezotto, T., Carvalho, R.F., Alves, L.R., Peters, L.J., Azevedo, R.A., 2015. Cadmium stress antioxidant responses and root-to-shoot communication in grafted tomato plants. Biometals 28, 803-816. doi:http://dx. doi.org/10.1007/s10534-015-9867-3.

Hänsch, R., Mendel, R.R., 2009. Physiological functions of mineral micronutrients (Cu, Zn, Mn, Fe, Ni, Mo, B, Cl). Curr. Opin. Plant Biol. 12, 259-266. doi:http://dx. doi.org/10.1016/j.pbi.2009.05.006.

Hédiji, H., Djebalia, W., Belkadhi, A., Cabasson, C., Moing, A., Rolin, D., Brouquisse, R., Gallusci, P., Chaïbi, W., 2015. Impact of long-term cadmium exposure on mineral content of Solanum lycopersicum plants: consequences on fruit production. S. Afr. J. Bot. 97, 176-181. doi:http://dx.doi.org/10.1016/j.sajb.2015.01.010.

He, J., Li, H., Luo, J., Ma, C., Li, S., Qu, L., Gai, Y., Jiang, X., Janz, D., Polle, A., Tyree, M., Luo, Z.-B., 2013a. A transcriptomic network underlies microstructural and physiological responses to cadmium in Populus $x$ canescens. Plant Physiol. 162 424-439.

He, J., Ma, C., Ma, Y., Li, H., Kang, J., Liu, T., Polle, A., Peng, C., Luo, Z.-B., 2013b. Cadmium tolerance in six poplar species. Environ. Sci. Pollut. Res. 20, 163-174. doi:http://dx.doi.org/10.1007/s11356-012-1008-8.

He, J., Li, H., Ma, C., Zhang, Y., Polle, A., Rennenberg, H., Cheng, X., Luo, Z.-B., 2015. Overexpression of bacterial c-glutamylcysteine synthetase mediates changes in cadmium influx, allocation and detoxification in poplar. New Phytol. 205, 240 254. doi:http://dx.doi.org/10.1111/nph.13013.

Heath, R.L., Packer, L., 1968. Photoperoxidation in isolated chloroplast. I. Kinetics and stoiichiometry of fatty acid peroxidation. Arch. Biochem. Biophys. 125, 189-198. doi:http://dx.doi.org/10.1016/0003-9861(68)90654-1. 
Hippler, F.W.R., Boaretto, R.M., Quaggio, J.A., Azevedo, R.A., Mattos, J.R.D., 2015. Towards soil management with $\mathrm{Zn}$ and Mn: estimates of fertilisation efficacy of Citrus trees. Ann. Appl. Biol. 166, 484-495. doi:http://dx.doi.org/10.1111/ aab.12197.

Iakimova, E.T., Woltering, E.J., Kapchina-Toteva, V.M., Frans, J.M., Harren, C., Simona, M.C., 2008. Cadmium toxicity in cultured tomato cells - role of ethylene, proteases and oxidative stress in cell death signaling. Cell Biol. Int. 32, 15211529. doi:http://dx.doi.org/10.1016/j.cellbi.2008.08.021.

Iannone, M.F., Rosales, E.P., Groppa, M.D., Benavides, M.P., 2010. Reactive oxygen species formation and cell death in catalase-deficient tobacco leaf disks exposed to cadmium. Protoplasma 245, 15-27. doi:http://dx.doi.org/10.1007/ s00709-009-0097-9.

Islam, E., Khan, M.T., Irem, S., 2015. Biochemical mechanisms of signaling: perspectives in plants under arsenic stress. Ecotoxicol. Environ. Saf. 114, 126133. doi:http://dx.doi.org/10.1016/j.ecoenv.2015.01.017.

Ivanchenko, M.G., Zhu, J., Wang, B., Medvecka', E., Du, Y., Azzarello, E., Mancuso, S., Megraw, M., Filichkin, S., Dubrovsky, J.G., Friml, J., Geisler, M., 2015. The cyclophilin A DIAGEOTROPICA gene affects auxin transport in both root and shoot to control lateral root formation. Development 142,1-10. doi:http://dx. doi.org/10.1242/dev.113225.

Jozefczak, M., Keunen, E., Schat, H., Bliek, M., Hernandez, L.E., Carleer, R., Remans, T., Bohler, S., Vangronsveld, J., Cuypers, A., 2014. Differential response of Arabidopsis leaves and roots to cadmium: glutathione-related chelating capacity vs antioxidant capacity. Plant Physiol. Biochem. 83, 1-9. doi:http://dx. doi.org/10.1016/j.plaphy.2014.07.001.

Kapoor, D., Kaur, S., Bhardwaj, R., 2014. Physiological and biochemical changes in Brassica juncea plants under Cd-induced stress. BioMed Res. Int. doi:http://dx. doi.org/10.1155/2014/726070.

Kelly, M.O., Bradford, K.J., 1986. Insensitivity of the diageotropica tomato mutant to auxin. Plant Physiol. 82, 713-717.

Keunen, E., Schellingen, K., Vangronsveld, J., Cuypers, A., 2016. Ethylene and metal stress: small molecule, big impact. Frontiens Plant Sci. doi:http://dx.doi.org/ 10.3389/fpls.2016.00023.

Khaliq, A., Zia-ul-Haq, M., Ali, F., Aslam, F., Matloob, A., Navab, A., Hussain, S., 2015. Salinity tolerance in wheat cultivars is related to enhanced activities of enzymatic antioxidants and reduced lipid peroxidation. Clean-Soil Air Water 43, 1248-1258. doi:http://dx.doi.org/10.1002/clen.201400854.

Koornneef, M., Bosma, T.D.G., Hanhart, C.J., Van der Veen, J.H., Zeevart, J.A.D., 1990. The isolation and characterization of gibberellin-deficient mutants in tomato. Theor. Appl. Genet. doi:http://dx.doi.org/10.1007/bf00224204.

Krishnamurthy, A., Rathinasabapathi, B., 2013. Auxin and its transport play a role in plant tolerance to arsenite-induced oxidative stress in Arabidopsis thaliana. Plant Cell Environ. 36, 1838-1849. doi:http://dx.doi.org/10.1111/pce.12093.

Lanahan, M.B., Yan, H.C., Giovannoni, J.J., Klee, H.J., 1994. The Never ripe mutation blocks ethylene perception in tomato. Plant Cell 6, 521-530.

Lichtentlaler, H.K., 1987. Chlorophylls and carotenoids: pigments of photosynthetic biomembranes. Methods Enzymol.148, 350-382. doi:http://dx.doi.org/10.1016/ 0076-6879(87)48036-1.

Liu, S.L., Yang, R.J., Ma, M.D., Dan, F., Zhao, Y., Jiang, P., Wang, M.H., 2015. Effects of exogenous NO on growth, mineral nutrient content, antioxidant system and ATPase activities of Trifoliumrepens L. plants under cadmium stress. Acta Physiologiae Plant. doi:http://dx.doi.org/10.1007/s11738-014-1721-7.

Luo, Z.-B., He, J., Polle, A., Rennenberg, H., 2016. Heavy metal accumulation and signal transduction in herbaceous and woody plants: paving the way for enhancing phytoremediation efficiency. Biotechnol. Adv. doi:http://dx.doi.org/ 10.1016/j.biotechadv.2016.07.003.

Lux, A., Martinka, M., Vaculík, M., White, P.J., 2011. Root responses to cadmium in the rhizosphere: a review. J. Exp. Bot. 62, 21-37. doi:http://dx.doi.org/10.1093/jxb/ erq281.

Lysenko, E., Klaus, A.A., Pshybytko, N.L., Kusnetsov, V.V., 2015. Cadmium accumulation in chloroplasts and its impact on chloroplastic processes in barley and maize. Photosynth. Res. 125, 291-303. doi:http://dx.doi.org/10.1007/ s11120-014-0047-z.

Melnyk, C.W., Schuster, C., Leyser, O., Meyerowitz, E.M., 2015. A developmental framework for graft formation and vascular reconnection in Arabidopsis thaliana. Curr. Biol. 25, 1306-1318. doi:http://dx.doi.org/10.1016/j. cub.2015.03.032.

Molnar, A., Melnyk, C.W., Bassett, A., Hardcastle, T.L., Dunn, R., Baulcombe, D.C., 2010. Small silencing RNAs in plants are mobile and direct epigenetic modification in recipients cells. Science 328, 872-875. doi:http://dx.doi.org/ 10.1126/science.1187959.

Moniuszko, G., 2015. Ethylene signaling pathway is not linear, however its lateral part is responsible for sensing and signaling of sulfur status in plants. Plant Signal Behav. doi:http://dx.doi.org/10.1080/15592324.2015.1067742.

Monteiro, C.C., Carvalho, R.F., Gratão, P.L., Carvalho, G., Tezotto, T., Medici, L.O., Peres, L.E.P., Azevedo, R.A., 2011. Biochemical responses of ethylene-insensitive Never ripe tomato mutant subjected to cadmium and salt stresses. Environ. Exp. Bot. 71, 306-320. doi:http://dx.doi.org/10.1016/j.envexpbot.2010.12.020.

Monteiro, C.C., Rolão, M.B., Franco, M.R., Peters, L.P., Cia, M.C., Capaldi, F.R., Carvalho, R.F., Gratão, P.L., Rossi, M.L., Martinelli, A.P., Peres, L.E.P., Azevedo, R.A., 2012. Biochemical and histological characterization of tomato mutants. An. Acad. Bras. Cienc. 84, 573-585.

Muradoglu, F., Gundogdu, M., Ercisli, S., Encu, T., Balta, F., Jaafar, H.Z.E., Muhammad, Z., 2015. Cadmium toxicity affects chlorophyll a and b content, antioxidant enzyme activities and mineral nutrient accumulation in strawberry. Biol. Res. doi:http://dx.doi.org/10.1186/s40659-015-0001-3.
Noctor, G., Foyer, C.H., 2016. Intracellular redox compartmentation and ROS-related communication in regulation and signaling. Plant Physiol. 171, 1581-1592. doi: http://dx.doi.org/10.1104/pp.16.00346;v.171 (p.1581-1592).

Nogueirol, R.C., Monteiro, F.A., Gratão, P.L., Borgo, L., Azevedo, R.A., 2015. Tropical soils with high aluminum concentrations cause oxidative stress in two tomato genotypes. Environ. Monit. Assess. 187, 73. doi:http://dx.doi.org/10.1007/ s10661-015-4282-3.

Omid, A., Keilin, T., Glass, A., Leshkowitz, D., Wolf, S., 2007. Characterization of phloem-sap transcription profile in melon plants. J. Exp. Bot. 58, 3645-3656. doi:http://dx.doi.org/10.1093/jxb/erm214.

Park, J.E., Park, J.Y., Kim, Y.S., Staswick, P.E., Jeon, J., Yun, J., Kim, S.Y., Kim, J., Lee, Y.H. Park, C.M., 2007. GH3-mediated auxin homeostasis links growth regulation with stress adaptation response in Arabidopsis. J. Biol. Chem. 282, 1003610046. doi:http://dx.doi.org/10.1074/jbc.M610524200.

Pereira, G.J.G., Molina, S.M.G., Lea, P.J., Azevedo, R.A., 2002. Activity of antioxidant enzymes in response to cadmium in Crotalaria juncea. Plant Soil 239, 123-132.

Piwowarczyk, B., Tokarz, K., Kamińska, I., 2016. Responses of grass pea seedlings to salinity stress in vitro culture conditions. Plant Cell, Tissue and Organ Culture 124, 227-240. doi:http://dx.doi.org/10.1007/s11240-015-0887-z.

Polle, A., Klein, T., Kettner, C., 2013. Impact of cadmium on young plants of Populus euphratica and P. x canescens, two poplar species that differ in stress tolerance. New For. 44,13-22. doi:http://dx.doi.org/10.1007/s11056-011-9301-9.

Pompeu, G.B., Vilhena, M.B., Gratão, P.L., Carvalho, R.F., Rossi, M.L., Martinelli, A.P. Azevedo, R.A., 2017. Abscisic acid-deficient sit tomato mutant responses to cadmium-induced stress. Protoplasma doi:http://dx.doi.org/10.1007/s00709016-0989-4 (in press.

Potters, G., Pasternak, T., Guisez, Y., Jansen, M.K., 2009. Different stresses, similar morphogenic responses: integrating a plethora of pathways. Plant Cell Environ 32, 158-169. doi:http://dx.doi.org/10.1111/j. 1365-3040.2008.01908.x.

Retzer, K., Luschnig, C., 2015. DIAGEOTROPICA: news from the auxin swamp. Trends Plant Sci. 20, 328-329. doi:http://dx.doi.org/10.1016/j.tplants (2015.04.009).

Rodríguez-Serrano, M., Romero-Puertas, M.C., Zabalza, A., Corpas, F.J., Gómez, M. del Río, L.A., Sandalio, L.M., 2006. Cadmium effect on oxidative metabolism of pea (Pisum sativum L.) roots: imaging of reactive oxygen species and nitric oxide accumulation in vivo. Plant Cell Environ. 29, 1532-1544. doi:http://dx.doi.org/ 10.1111/j. 1365-3040.2006.01531.x.

Romero-Puertas, M.C., Corpas, F.J., Rodríguez-Serrano, M., Gomez, M., delRío, L.A. Sandalio, L.M., 2007. Differential expression and regulation of antioxidative enzymes by cadmium in pea plants. J. Plant Physiol. 164, 1346-1357. doi:http:// dx.doi.org/10.1016/j.jplph.2006.06.018.

Roychoudhury, A., Basu, S., Sengupta, D.N., 2012. Antioxidants and stress-related metabolites in the seedlings of two indica rice varieties exposed to cadmium chloride toxicity. Acta Physiologiae Plant. 34, 835-847. doi:http://dx.doi.org/ 10.1007/s11738-011-0881-y.

Schellingen, K., Van Der Straeten, D., Vandenbussche, F., Prinsen, E., Remans, T., Vangronsveld, J., Cuypers, A., 2014. Cadmium-induced ethylene production and responses in Arabidopsis thaliana rely on ACS2 and ACS6 gene expression. BMC Plant Biol. 14, 214. doi:http://dx.doi.org/10.1186/s12870-014-0214-6.

Schellingen, K., Van Der Straeten, D., Remans, T., Vangronsveld, J., Keunena, E., Cuypers, A., 2015. Ethylene signalling is mediating the early cadmium-induced oxidative challenge in Arabidopsis thaliana. Plant Sci. 239, 137-146. doi:http:// dx.doi.org/10.1016/j.plantsci.2015.07.015.

Seregin, I.V., Kozhevnikova, A.D., 2006. Physiological role of nickel and its toxic effects on higher plants. Russ. J. Plant Physiol. 53, 257-277. doi:http://dx.doi. org/10.1134/S1021443706020178.

Shabala, S., White, R.G., Djordjevic, M.A., Ruan, Y.-L., Mathesius, U., 2016. Root-toshoot signalling: integration of diverse molecules, pathways and functions. Funct. Plant Biol. 43, 87-104. doi:http://dx.doi.org/10.1071/FP15252.

Shafi, M., Guoping, Z., Bakht, J., Khan, M.A., Ejaz-ul, I., Khan, M.D., Raziuddin, 2010. Effect of cadmium and salinity stresses on root morphology of wheat. Pak. J. Bot. $42,2747-2754$

Sharma, S.S., Dietz, K.-J., 2009. The relationship between metal toxicity and cellular redox imbalance. Trends Plant Sci. 14, 43-50. doi:http://dx.doi.org/10.1016/j. tplants.2008.10.007.

Shi, W.-G., Li, H., Liu, T.-X., Polle, A., Peng, C.-H., Luo, Z.-B., 2015. Exogenous abscisic acid alleviates zinc uptake and accumulation in Populus $\times$ canescens exposed to excess zinc. Plant Cell Environ. 38, 207-223. doi:http://dx.doi.org/10.1111/ pce.12434.

Siedlecka, A., Krupa, Z., 1999. Cd/Fe interaction in higher plants-its consequences for the photosynthetic apparatus. Photosynthetica 36, 321-331. doi:http://dx. doi.org/10.1023/A:1007097518297.

Spiegelman, Z., Golan, G., Wolf, S., 2013. Don't kill the messenger: long-distance trafficking of mRNA molecules. Plant Sci. 213, 1-8. doi:http://dx.doi.org/ 10.1016/j.plantsci.2013.08.011.

Talukdar, D., 2011. Isolation and characterization of $\mathrm{NaCl}$-tolerant mutations in two important legumes, Clitoria ternatea L. and Lathyrus sativus L: : induced mutagenesis and selection by salt stress. J.Med. Plants Res. 5, 3619-3628.

Tezotto, T., Favarin, J.L., Neto, A.P., Gratão, P.L., Azevedo, R.A., Mazzafera, P., 2013. Simple procedure for nutrient analysis of coffee plant with energy dispersive Xray fluorescence spectrometry (EDXRF). Sci. Agr. 70, 263-267. doi:http://dx.doi. org/10.1590/S0103-90162013000400007.

Tognetti, V.B., Mühlenbock, P., Van Breusegem, F., 2011. Stress homeostasis -the redox and auxin perspective. Plant Cell Environ. 35, 321-333. doi:http://dx.doi. org/10.1111/j. 1365-3040.2011.02324.x.

Uraguchi, S., Mori, S., Kuramata, M., Kawasaki, A., Arao, T., Ishikawa, S., 2009. Rootto-shoot Cd translocation via the xylem is the major process determining shoot 
and grain cadmium accumulation in rice. J. Exp. Bot. 60, 2677-2688. doi:http:// dx.doi.org/10.1093/jxb/erp119.

Van de Poel, B., Smet, D., Van Der Straeten, D., 2015. Ethylene and hormonal crosstalk in vegetative growth and development. Plant Physiol. 169, 61-72. doi: http://dx.doi.org/10.1104/pp.15.00724.

Vincent, J.M., 1975. Manual Practico De Rizobiologia, 1 st ed. Hemisferio Sur, Buenos Aires.

Wilkinson, J.Q., Lanahan, M.B., Yen, H.-C., Giovannoni, J.J., Klee, H.J., 1995. An ethylene inducible component of signal transduction encoded by Never-ripe. Science 270, 1807-1809. doi:http://dx.doi.org/10.1126/science.270.5243.1807.

Wu, F., Zhang, G., Dominy, P., Wu, H., Bachir, D.M.L., 2007. Differences in yield components and kernel $\mathrm{Cd}$ accumulation in response to $\mathrm{Cd}$ toxicity in four barley genotypes. Chemosphere 70, 83-92. doi:http://dx.doi.org/10.1016/j. chemosphere.2007.06.051.

Wu, Z., Zhao, X., Sun, X., Tan, Q., Tang, Y., Nie, Z., Qu, C., Chen, Z., Hu, C., 2015. Antioxidant enzyme systems and the ascorbate-glutathione cycle as contributing factors to cadmium accumulation and tolerance in two oilseed rape cultivars (Brassica napus L.) under moderate cadmium stress. Chemosphere 138, 526-536. doi:http://dx.doi.org/10.1016/j.chemosphere.2015.06.080.

Zhang, H.Z., Guo, Q.J., Yang, J.X., Chen, T., Zhu, G., Peters, M., Wei, R., Tian, L., Wang, C., Tan, D. Ma, J., Wang, G. Wan, Y., 2014. Cadmium accumulation and tolerance of two castor cultivars in relation to antioxidant systems. J. Environ. Sci. 26, 20482055. doi:http://dx.doi.org/10.1016/j.jes.2014.08.005.

Zouari, M., Ahmed, C.B., Elloumi, N., Bellassoued, K., Delmail, D., Labrousse, P., Abdallah, F.B., Rouina, B.B., 2016a. Impact of proline application on cadmium accumulation, mineral nutrition and enzymatic antioxidant defence system of Oleaeuropaea L. cv Chemlali exposed to cadmium stress. Ecotoxicol. Environ. Saf. 128, 195-205. doi:http://dx.doi.org/10.1016/j.ecoenv.2016.02.024.

Zouari, M., Elloumi, N., Ahmed, C.B., Delmail, D., Rouina, B.B., Abdallah, F.B., Labrousse, P., 2016b. Exogenous proline enhances growth, mineral uptake, antioxidant defence and reduces cadmium-induced oxidative damage in young date palm (Phoenix dactylifera L.). Ecol. Eng. 86, 202-209. doi:http://dx.doi.org/ 10.1016/j.ecoleng.2015.11.016. 